\title{
Interplay of diffusion and dissociation mechanisms during hydrogen absorption in metals
}

\author{
A. Borgschulte* and R. Gremaud \\ EMPA Swiss Federal Laboratories for Materials Testing and Research, Laboratory 138 (Hydrogen \& Energy), Überlandstrasse 129, \\ CH-8600 Dübendorf, Switzerland \\ R. Griessen \\ Division of Physics and Astronomy, Faculty of Sciences, Vrije Universiteit, De Boelelaan 1081, 1081 HV Amsterdam, The Netherlands
}

(Received 24 September 2007; revised manuscript received 3 June 2008; published 16 September 2008)

\begin{abstract}
Kinetic measurements of gas-solid reactions, in particular hydrogen-sorption kinetics, are usually interpreted according to single rate-limiting step models. However, recent studies gave clear evidence for the interdependence of fundamentally different steps involved in hydrogen sorption. This interdependence is explored in a one-dimensional continuum model in order to estimate the time, temperature, and pressure dependences of the sorption kinetics and the relevance of several materials parameters involved in the process. Quantitative descriptions of the extraction of physical parameters for various scenarios are given. The model is successfully tested for several experimental cases, ranging from model systems (thin films) to practical systems $\left(\mathrm{LaNi}_{5} \mathrm{H}_{x}\right.$ and $\mathrm{MgH}_{2}$ powder samples).
\end{abstract}

DOI: 10.1103/PhysRevB.78.094106 PACS number(s): 64.60.-i, 05.50.+q, 05.70.-a, 82.20. $-\mathrm{w}$

\section{INTRODUCTION}

The search for novel hydrogen storage materials has triggered the investigation of the interaction of hydrogen with hundreds of materials. ${ }^{1}$ As the main interest often lies in the technical applicability of specific materials for hydrogen storage, the emphasis is usually not put on well-defined determination of fundamental physical parameters. This complicates the comparison of published experimental results, since the data depend often on specific details of the followed experimental procedures. A typical example of such a situation is found for $\mathrm{LaNi}_{5} \mathrm{H}_{6}$, a hydrogen storage material with appropriate thermodynamics and sufficiently fast kinetics. The physical origin of its activation process has intensively been investigated, in particular the influence of the preparation method on the $\mathrm{H}$-sorption kinetics (see, e.g., Refs. 2-7 and references therein). On the fundamental side, kinetic barriers such as dissociation and diffusion barriers were calculated by ab initio models (see, e.g., Refs. 8-11). Due to uncertainties in model and experimental parameters, only the calculated activation energies of such barriers are compared to experimentally derived values. Moreover this is done with relatively small success. An enlightening analysis of this problem is in the paper of Andreasen et al. ${ }^{12}$ Assuming an Arrhenius behavior with rate $R$,

$$
R=R_{0} e^{-E_{\mathrm{AA}} / k T},
$$

they compared the activation energies derived from published experimental results on the kinetics of $\mathrm{LaNi}_{5} \mathrm{H}_{6}$. These energies, which we are calling from now on "apparent activation energies" $E_{\mathrm{AA}}$, range from less than $0.2 \mathrm{eV}$ to more than $0.5 \mathrm{eV}$. Simultaneously, the logarithm of the prefactor $\ln R_{0}$ scales linearly with $E_{\mathrm{AA}}$, so as to compensate for the influence of the activation energy. This leads to the so-called Constable-Cremer relation ${ }^{13}$ or compensation effect, ${ }^{14}$ as coined by catalysis research. ${ }^{15}$ The underlying problem originates from the fact that the Arrhenius equation [Eq. (1)] neglects the fact that the initial and final states, which are sepa- rated by an energy barrier, can change. This, on the other hand, is the principal basis of hydrogen storage in metal hydrides, where the rate (i.e., uptake or release of hydrogen) is determined by the difference between the chemical potentials of hydrogen in the gas phase and in the metal hydride. The thermodynamic driving force results from the gradient of the chemical potential $\frac{\partial \mu}{\partial x}$, and the rate is given as

$$
R=-£ \frac{\partial \mu}{\partial x},
$$

as demanded by Onsager's kinetic theory. ${ }^{16} £$ is a phenomenological coefficient, which depends on temperature and concentration. The activation energy of the process is included in $£ . \mu$ is temperature dependent, and thus an Arrhenius analysis is difficult. Equation (2) can be solved analytically only for simple cases, e.g., binary diffusion, giving Fick's law. In complex systems, we have to consider various $\mu$ 's and different $£$ 's. Due to the multiplicity of processes (surface, subsurface, intermediate layers, etc.), ${ }^{17}$ neither an analytical solution of Eq. (2) is possible nor are all mandatory fundamental parameters known (e.g., coverage, energy barriers, and thermodynamic parameters), which would allow for a numerical solution.

In this paper we develop a simplified two-step model of the hydrogen-sorption kinetics in metal hydrides considering dissociation and diffusion. The basis of the calculation is relation (2), which is specified to fit the processes. The obtained differential equations are approximated to allow for an analytical solution, from which the time, temperature, and pressure dependences of absorption and desorption kinetics and the influence of several materials parameters involved in the process are derived. The central result of this paper is that the kinetics can hardly be described by a single rate-limiting step. The kinetics is in most cases the result of the interplay of dissociation and diffusion. Very similar to heterogeneous catalysis, the observed compensation effect can be explained by the interplay of these two processes. The model is suc- 
cessfully tested for several experimental cases, ranging from model systems (thin films) to practical systems $\left(\mathrm{LaNi}_{5} \mathrm{H}_{x}\right.$ and $\mathrm{MgH}_{2}$ ). Particular emphasis is laid on the calculation of the apparent activation energy, which depends now on temperature and pressure. These dependencies complicate the correct interpretation of the measured activation energy.

\section{MODEL}

\section{A. Basic assumptions}

We consider a system consisting of a hydrogen absorbing metal covered by a surface layer as sketched in Fig. 1. Hydrogen enters the metal through the top layer and forms the metal hydride via several intermediate steps, which have characteristic energy parameters (activation energies $E_{i}$ are defined as the difference between initial and final states; enthalpies $\Delta H_{i}$ are relative to the gas phase potential at normal conditions.):

(1) physisorption by molecular hydrogen: $\mathrm{H}_{2}^{\text {gas }} \rightleftharpoons \mathrm{H}_{2}^{\text {phys }}$, with heat of physisorption $\Delta H_{\text {phys }} \leq-0.05 \mathrm{eV} / \mathrm{H}_{2}$.

(2) dissociation and chemisorption of atomic hydrogen (coverage of the surface $\left.\theta_{\mathrm{H}}\right) \theta: \mathrm{H}_{2}^{\text {phys }} \rightleftharpoons 2 \mathrm{H}^{\text {chem }}$, with dissociation barrier height $E_{\mathrm{diss}}=0-2 \mathrm{eV}$ and heat of chemisorption $\Delta H_{\text {chem }}=-0.1$ to $-2 \mathrm{eV} / \mathrm{H}$.

(3) surface penetration: $\mathrm{H}^{\text {chem }} \rightleftharpoons \mathrm{H}^{\text {sub }}$, with activation barrier height $E_{\text {sub }}$ and enthalpy $\Delta H_{\text {sub }}=-0.25$ to $1.25 \mathrm{eV}$.

(4) diffusion through surface layer, with hydrogen concentration $c(0<x<L)$, activation energy for diffusion $E_{\text {diff }}$ $\simeq 0.5 \mathrm{eV}, \quad$ and heat of solubility $\Delta H_{\mathrm{sol}}=-0.2$ to $+0.2 \mathrm{eV} / \mathrm{H}$.

(5) diffusion through metal: $\mathrm{H}_{\text {sol }}^{\text {metal }}\left(x_{3}\right) \rightleftharpoons \mathrm{H}_{\text {sol }}^{\text {metal }}\left(x_{4}\right)$, with hydrogen concentration $c_{\mathrm{MH}}(x>L)$, activation energy for diffusion $E_{\text {diff }}^{\text {metal }} \leq 0.2 \mathrm{eV}$, and heat of solubility $\Delta H_{\text {sol }}^{\text {metal }}=$ +0.2 to $-0.2 \mathrm{eV} / \mathrm{H}$.

(6) hydride formation: $\mathrm{H}_{\text {sol }}^{\text {metal }} \rightleftharpoons \mathrm{H}_{\mathrm{MH}}$, with heat of formation $\Delta H_{\mathrm{MH}} \simeq-0.5 \mathrm{eV} / \mathrm{H}$.

This discussion lists most of the mechanisms involved in hydrogen sorption. It is noted, though, that a full description requires a quantum-mechanical treatment of the processes. ${ }^{18}$ It is not practical to consider all the steps involved in hydrogen sorption. We can however already gain valuable insight from a simple model involving one surface barrier, one diffusion barrier, and one constant hydrogen reservoir. In the following we give some arguments to underline the validity of this simplification for many relevant hydride systems.

Since the physisorption of gas molecules on surfaces requires almost no activation energy and the impingement rate of $\mathrm{H}_{2}$ onto the surface is high, the gas phase can be assumed to be in equilibrium with the physisorbed state. Furthermore, the heat of formation $\Delta H_{\text {phys }}$ is very small and the molecule remains in its integrity. This step is thus neglected in our model. For chemisorption, the hydrogen molecule has to split, and for that it has to overcome in most cases a high dissociation barrier of $0 \leq \Delta E_{\text {diss }}<2 \mathrm{eV}$. Hydrogen is relatively strongly bound to metal surfaces $\left(\Delta H_{\text {chem }} \simeq\right.$ $-1 \mathrm{eV} / \mathrm{H})$. Thus, the coverage $\theta$ is high under technical conditions. (At hydrogen pressures of 1-100 bars and 300-500 $\mathrm{K}$, it varies between 0.6 and 0.9 for transition metals. ${ }^{19}$ ) The hydrogen coverage on oxides is in the same range as found

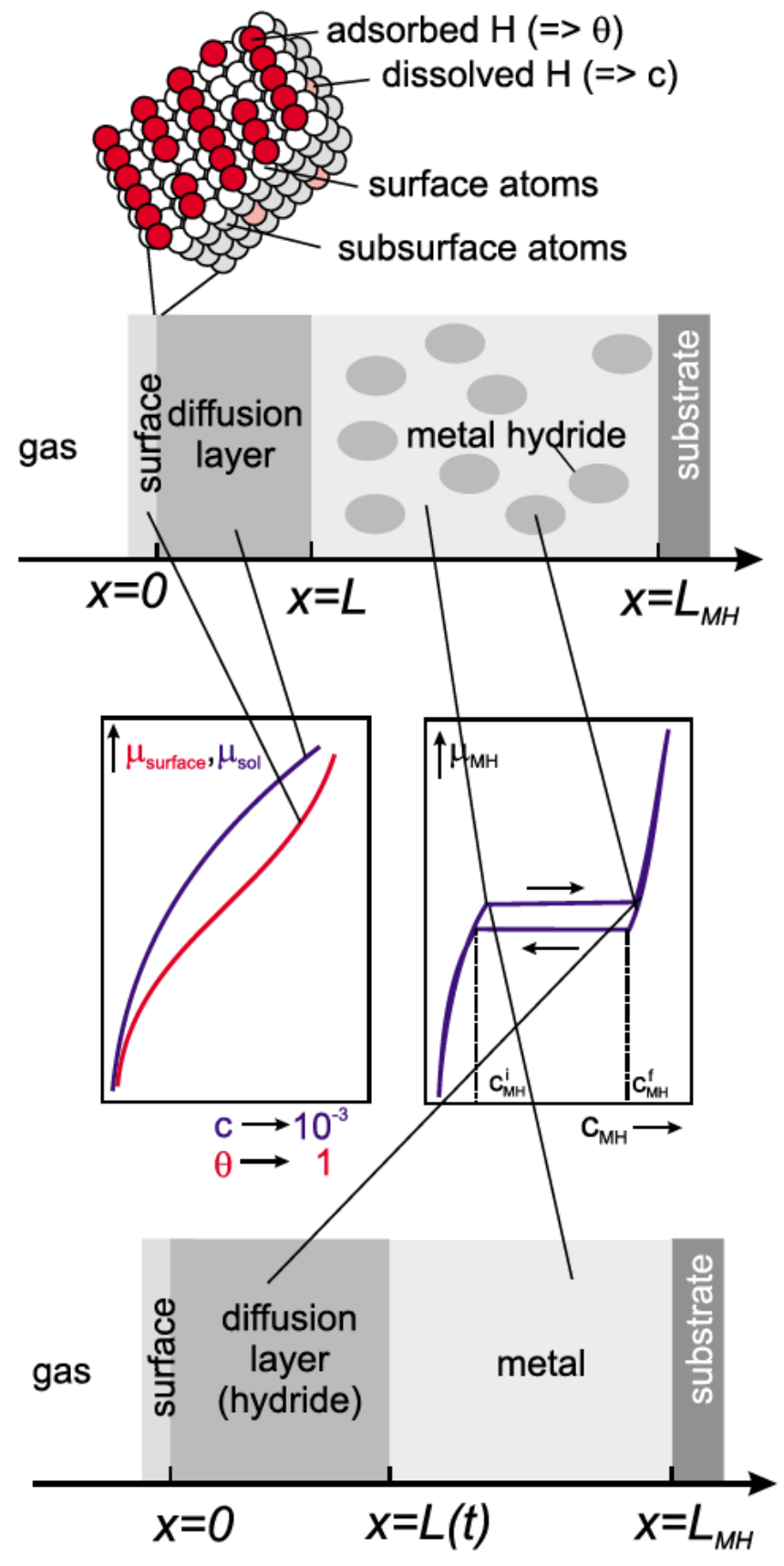

FIG. 1. (Color online) Top and bottom panels: The various layers considered in the model. The surface layer consists of hydrogen adsorbed on surface atoms (amount of $\mathrm{H}$ is described by $\theta$ ) attached to subsurface atoms, which belong to the diffusion layer. The hydrogen concentration in the diffusion layer is labeled $c$; the concentration in the metal hydride is $c_{\mathrm{MH}}$. Two typical situations are sketched: The top panel shows a metal hydride with fast diffusion inside the metal/metal hydride, where the metal hydride nucleates homogeneously. The main diffusion barrier is the surface. The bottom panel sketches the formation of a hydride with a very slow diffusion of hydrogen in the metal hydride. The main diffusion barrier is the metal hydride. Middle panel: Schematic representation of the concentration dependence of the hydrogen chemical potential in (left) the diffusion layer and (right) in the metal hydride for a given temperature. 


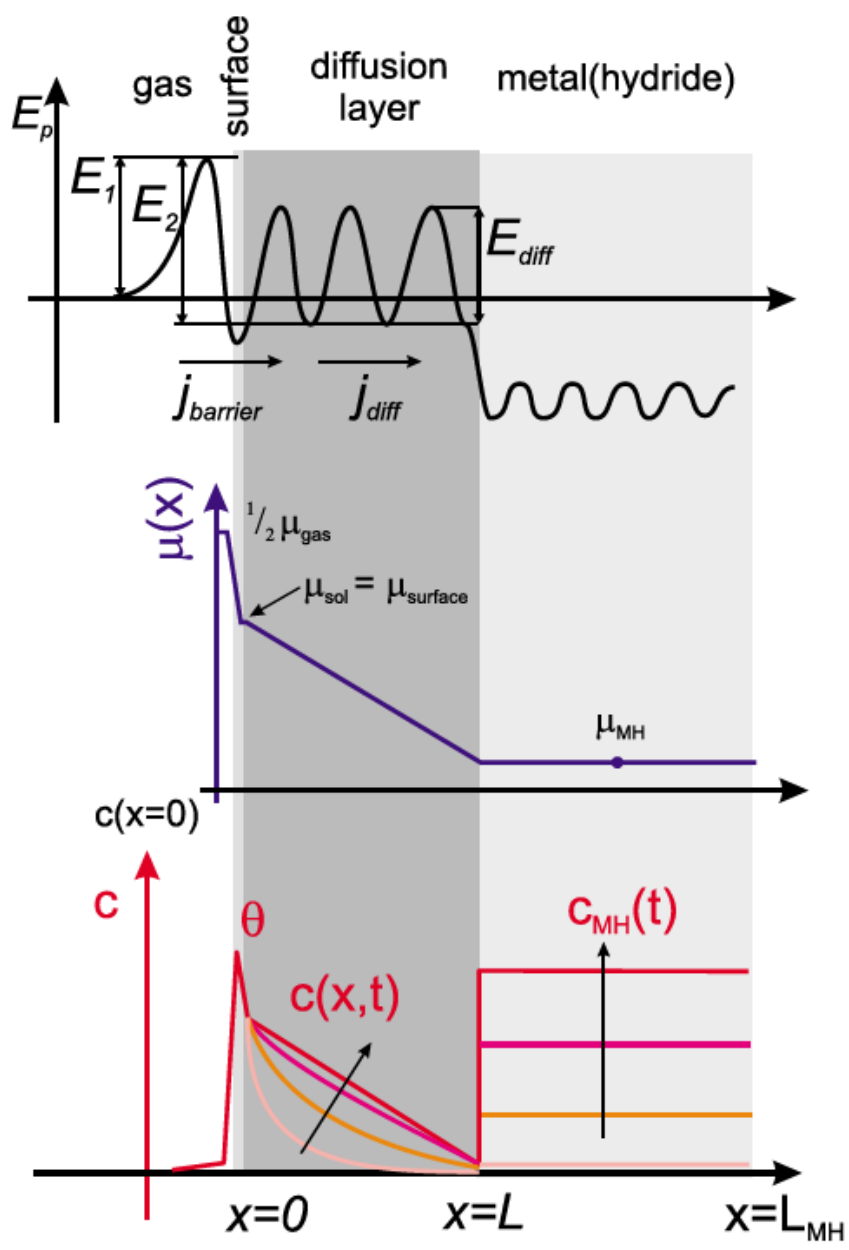

FIG. 2. (Color online) Scheme of the two-step model used to define the variables used in this work. Top, middle, and bottom panels: Potential energy, chemical potential, and hydrogen concentration as a function of the location in the diffusion layer and in the metal hydride, considering also the inherent hysteresis of the $p-c$ isotherms of any metal hydride. In the metal (hydride) the diffusion is $\infty$. Therefore, the concentration of hydrogen is determined by the current $j_{\text {diff }}(x=L, t)$. The diffusion current $j_{\text {diff }}(x=0, t)$ is equal to the current $j_{\text {barrier }}$ through the surface barrier with height $E_{1}$.

experimentally. ${ }^{20}$ The next step is the hopping of $\mathrm{H}$ from a surface site to a subsurface site. The corresponding energy barriers are $\Delta E_{\text {sub }}-\Delta H_{\text {chem }}$ for absorption and $\Delta E_{\text {sub }}-\Delta H_{\text {sub }}$ for desorption. The moving species is atomic hydrogen and thus this step can be considered as a diffusion process between two phases (surface and subsurface). Furthermore, as the heat of hydrogen solution in the subsurface layer is similar to that in the following layers, i.e., $\Delta H_{\text {sub }} \simeq \Delta H_{\text {sol }}$, and $\Delta E_{\text {sub }} \simeq \Delta E_{\text {diff, }}$, we equate the subsurface hydrogen with "regularly" dissolved hydrogen in the surface layer, i.e., steps 3 and 4 are modeled by one diffusion process between the surface and bulk hydrogen. This diffusion barrier can be an oxide skin and/or diffusion in the metal, in which the metal hydride nucleates and grows ("diffusion layer" in Fig. 2). In most metals, hydrogen diffusion is fast. Thus our model calculations considers only one (averaged) diffusion barrier. A prerequisite of our model is that the diffusion parameters (e.g., diffusion layer thickness) are constant or their change is slow compared to the other processes (quasiequilibrium). The metal and randomly distributed metal-hydride nuclei are assumed to be in thermodynamic equilibrium ("hydride plateau"), which is mainly determined by the heat of hydride formation $\Delta H_{\mathrm{MH}}$. Furthermore, the supersaturation required for hydride formation/decomposition is assumed to be small (corresponds to a small hysteresis; see later discussion). If it is substantial, the hydride growth mode will change, e.g., in hydrogen absorption in $\mathrm{Mg}$. Here, a hydride layer on top of the metal is formed, which grows on the expense of the metal as the reaction proceeds (see Fig. 2). ${ }^{21}$ This special growth mode can still be treated with the two-step model if instead of considering a constant surface layer thickness, an increasing diffusion layer thickness is included.

To summarize, the two-step model includes a surface layer with a dissociation barrier $\left(E_{1}\right.$, corresponding to $\left.E_{\text {diss }}\right)$ and a diffusion layer with a diffusion barrier $E_{\text {diff. }}$ After dissociation at the surface (near $x=0$ ), hydrogen passes through a subsurface site and diffuses through the diffusion layer (binding energy $E_{1}-E_{2}$, corresponding to $2 \Delta H_{\text {sol }}$ ) to enter the hydride at $x=L$. The corresponding energy potential is drawn in the top panel of Fig. 2. The energy minima represent stable states of hydrogen in matter, i.e., chemisorbed hydrogen, hydrogen dissolved in the catalyst (diffusion layer), and hydrogen in the hydride. The hydrogen concentration profiles at various times during hydrogen loading are schematically indicated in the lower panel of Fig. 2. The chemical potential is continuous (see middle panel) even at $x=0$, at which it changes over a very short distance due to the dissociation barrier. The hydrogen concentration dependence of the chemical potential is sketched for the diffusion layer $(0<x<L)$ and the metal hydride in the middle panels of Fig. 2. The metal hydride is assumed to have a flat plateau at pressure $p_{\mathrm{pl}}$ for concentrations in the range $0 \simeq c_{\mathrm{MH}}^{i}$ $<c_{\mathrm{MH}}<c_{\mathrm{MH}}^{f} \simeq 1$. An additional difficulty, which is first neglected during derivation of the model, is the difference between the absorption plateau pressure and desorption plateau pressure in the isotherm, which leads to hysteresis effects in the metal hydride. We describe now the mathematics and simplifications of the two-step model.

\section{Particle current at the surface}

The net current $j_{\text {barrier }}$ consists of an inward current over the barrier $E_{1}$ and an outward current over the barrier $E_{2}$. The inward current is proportional to the density of $\mathrm{H}_{2}$ molecules at the surface and the fraction of unoccupied surface sites $(1-\theta)^{2}$ (at least two vacancies are needed), ${ }^{22}$ where $\theta$ is the fraction of surface sites occupied by atomic hydrogen. The outward current is proportional to $\theta^{2}$ since two hydrogen atoms have to recombine to form an $\mathrm{H}_{2}$ molecule.

$$
j_{\text {barrier }}=a p(1-\theta)^{2} e^{-E_{1} / k T}-b \theta^{2} e^{-E_{2} / k T} .
$$

The prefactors $a$ and $b$ are constants, which will be defined later. The surface barrier leads to a step in the chemical potential from hydrogen as a gas to hydrogen chemisorbed at the surface. Further, the chemical potential is assumed to be continuous. For simplification the concentration $c(x=0)$ in the diffusion layer is assumed to be in equilibrium with $\theta$. 


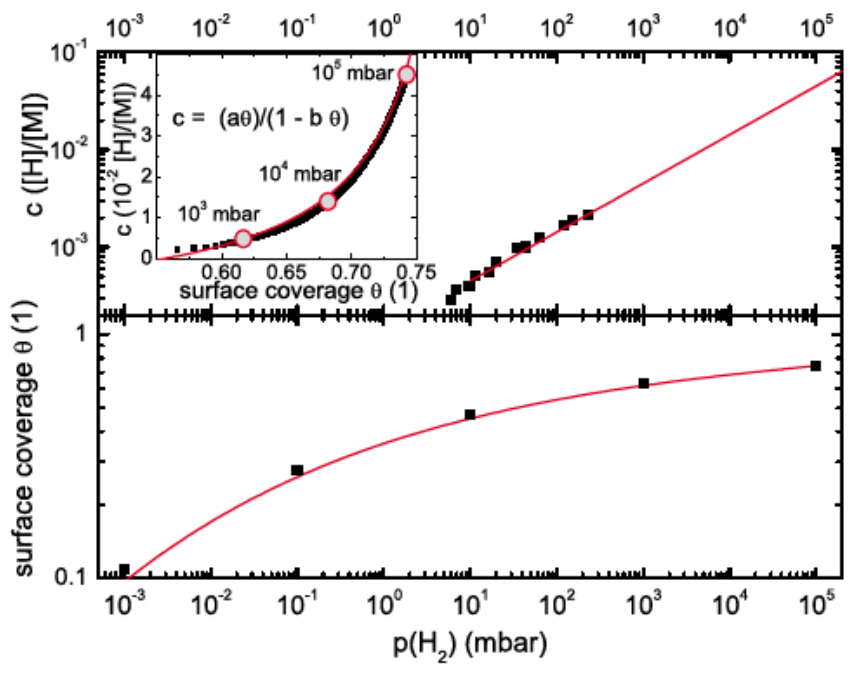

FIG. 3. (Color online) Pressure dependence at $600 \mathrm{~K}$ of the surface coverage (calculations from Ref. 19) and hydrogen bulk concentration (experimental values from Ref. 25) of Ni. The lines are extrapolations used to plot the bulk concentration as a function of the surface coverage (inset). A good fit (line) to these values is $c(\theta) \propto \theta /(1+a \theta)$, with $a \simeq-1.25$, in fair agreement with the used approximation [Eq. (5)].

The equality of the chemical potentials leads to the following relation between $\theta$ and $c$ (Ref. 23):

$$
\frac{\theta}{1-\theta} e^{-\mu_{\text {surface }} / k T}=\frac{c}{1-c} e^{-\mu_{\text {sol }} / k T} .
$$

$\mu_{\text {surface }}$ and $\mu_{\text {bulk }}$ are the chemical potentials of hydrogen, which are mainly determined by the binding energies of hydrogen on the surface, $\Delta H_{\text {chem }}$, and in the bulk, $\Delta H_{\text {sol }}$ (see Fig. 2).

In most cases, the binding energy of hydrogen is larger at the surface than in the bulk, ${ }^{24}$ and thus the surface concentration is larger, too (see bottom panel of Fig. 2). As we are interested only in situations where the concentration $c$ is much smaller than 1 , we take

$$
\frac{\theta}{1-\theta}=g c,
$$

with $g$ as the so-called surface enrichment factor: $g$ $\propto e^{\left(\mu_{\text {sol }}-\mu_{\text {surface }}\right) / k T}$. Equation (3) becomes

$$
j_{\text {barrier }}=(1-\theta)^{2}\left(a p e^{-E_{1} / k T}-b g^{2} c^{2} e^{-E_{2} / k T}\right) .
$$

The "self-blocking factor" $s^{2}=(1-\theta)^{2}$ is

$$
s^{2}=\left(1-\frac{g c}{1+g c}\right)^{2} .
$$

In many cases, $s^{2}$ can be assumed to be constant, which is equivalent to the effect that hydrogen is "trapped" on a surface due to the high negative heat of chemisorption (large $g$ ) and thus the coverage is high (for typical $3 d$ metals, $\theta$ $\geq 0.7$ at high pressures; ${ }^{19}$ see Fig. 3 ). The deviation from the equilibrium value of the self-blocking factor induced by a change in the bulk hydrogen concentration $c$ is therefore low.
The latter assumptions are critical, as they are indispensable for an analytical model on hand, but may oversimplify nature on the other hand. To underline the validity of the assumptions, we show the dependence of coverage and dissolved hydrogen in $\mathrm{Ni}$ on the applied pressure (Fig. 3). The coverage does not change much within the typical pressure range of 0.1-100 bars, while the amount of dissolved hydrogen changes drastically (Sievert's regime). The relation between coverage and hydrogen concentration is shown to be well approximated by Eq. (5).

\section{Diffusion current at $x=0$}

In dynamic equilibrium, the overbarrier current $j_{\text {barrier }}$ must be equal to the diffusion current $j_{\text {diff }}$ at beginning of the diffusion layer. Thus,

$$
j_{\text {barrier }}=j_{\text {diff }}(x=0, t)=-\left.£ \frac{\partial \mu}{\partial x}\right|_{x=0},
$$

where $\mu$ is the chemical potential of hydrogen in the diffusion layer. The coefficient $£$ depends on the hydrogen concentration since in the limit of low concentrations the diffusion current is given by Fick's law:

$$
j_{\text {diff }}=-D \rho \frac{d c}{d x},
$$

with $D$ as the constant diffusion coefficient and $\rho$ as the density of available hydrogen sites per volume (scaling of $c$ ). In addition, $£=£_{0}(1-c)$, since hydrogen can jump only to an unoccupied interstitial site. Assuming an ideal solution model with

$$
\mu=k T \ln \left(\frac{c}{(1-c)}\right)+\Delta H_{\mathrm{sol}},
$$

we obtain

$$
\frac{d \mu}{d c}=\frac{k T}{c(1-c)}
$$

and

$$
j_{\text {diff }}=-£ \frac{d \mu}{d x}=-£_{0}(1-c) \frac{d \mu}{d c} \frac{d c}{d x}=-£_{0} \frac{k T}{c} \frac{d c}{d x} .
$$

Consequently, after comparison with Fick's first law, $j=$ $-D \rho \frac{d c}{d x}$, we obtain

$$
£_{0}=\frac{c D \rho}{k T} \text { and } £=D \rho \frac{c(1-c)}{k T} \text {, }
$$

and we can rewrite Eq. (8) as

$$
j_{\text {barrier }}=j_{\text {diff }}(x=0, t)=-\left.D \rho \frac{\partial c}{\partial x}\right|_{x=0} .
$$

This is the standard diffusion equation. Note, however, that the chemical potential of hydrogen is continuous throughout the sample, while the concentration can have discontinuities, e.g., at $x=L$. 


\section{Diffusion current in the diffusion layer}

Fick's second law determines the diffusion in the diffusion layer $(0<x<L)$ :

$$
\frac{\partial c}{\partial t}=D \frac{\partial^{2} c}{\partial^{2} x}
$$

In the diffusion layer, hydrogen is bound with $E_{1}-E_{2}$ $\equiv 2 \Delta H_{\mathrm{sol}}$, where $\Delta H_{\mathrm{sol}}$ is the heat of hydrogen solution in the diffusion layer. ${ }^{26}$

\section{Concentration inside the metal hydride.}

The number of hydrogen per second and per $\mathrm{m}^{2}$ entering/ leaving the metal hydride $(\mathrm{MH})$ is

$$
j_{\text {diff }}(x=L, t)=\left(L_{\mathrm{MH}}-L\right) \rho \frac{d c_{\mathrm{MH}}}{d t},
$$

where $L_{\mathrm{MH}}-L$ is the thickness of the metal-hydride layer. Since the metal-hydride plateau is assumed to be flat from $c_{\mathrm{MH} i}$ to $c_{\mathrm{MH} f}$ [see the sketched $\mu\left(c_{\mathrm{MH}}\right)$ curves in Fig. 1] and the diffusion to be very rapid (i.e., $D_{\mathrm{MH}} \rightarrow \infty$ ), $c_{\mathrm{MH}}=$ const and the chemical potential $\mu_{\mathrm{MH}}$ is taken to be equal to a constant $\mu_{\mathrm{pl}}$. The continuity of the chemical potential at the interface between the diffusion layer and the metal hydride is

$$
\mu(x=L, t)=\mu_{\mathrm{pl}} .
$$

With this the concentration $c(x=L, t) \simeq c_{\mathrm{pl}}$ in the diffusion layer is related to the plateau pressure $p_{\mathrm{pl}}$ in metal hydride through

$$
k T \ln \left(\frac{c_{\mathrm{pl}}}{1-c_{\mathrm{pl}}}\right)+\Delta H_{\mathrm{sol}}-\frac{\Delta S_{0}}{k}=\frac{1}{2} k T \ln \left(\frac{p_{\mathrm{pl}}}{p_{0}}\right),
$$

where $p_{0}$ is the standard pressure and $\Delta S_{0}$ is the entropy change at standard conditions. As we assume that the metal hydride has relatively low-pressure plateaus, we are in the low-concentration limit in the diffusion layer and Sievert's law is valid [see the sketched $\mu\left(c_{H}\right)$ curves in Fig. 2]:

$$
c(x=L, t)=c_{\mathrm{pl}}=\alpha \sqrt{p_{\mathrm{pl}}},
$$

where $\alpha$ is Sievert's constant. The temperature dependence of $\alpha$ is $\alpha \propto \exp \left(-\Delta H_{\mathrm{sol}} / k T\right)$. At equilibrium, i.e., $j_{\text {barrier }}=0$, the concentration is the same everywhere in the whole diffusion layer, and in particular $c(x=0)=c_{\mathrm{pl}}$. With this we can correlate the bulk constant with the surface parameters via Eq. (6):

$$
\alpha=\sqrt{\frac{a}{b g^{2}}} \exp \left(\frac{E_{2}-E_{1}}{2 k T}\right) .
$$

\section{Summary}

Taking all the points mentioned above into consideration, we obtain the following:

(1) For the surface at $x=0$,

$$
\begin{gathered}
\left(1-\frac{g c}{1+g c}\right)^{2}\left(a e^{-E_{1} / k T} p-b g^{2} e^{-E_{2} / k T} c^{2}\right) \\
=-\left.D_{0} e^{-E_{\mathrm{diff}} / k T} \rho \frac{\partial c}{\partial x}\right|_{x=0} .
\end{gathered}
$$

$D_{0}$ is the prefactor of diffusion, $E_{\text {diff }}$ the diffusion barrier height (see Fig. 2).

(2) For the diffusion layer at $0<x<L$,

$$
\frac{\partial c}{\partial t}=D \frac{\partial^{2} c}{\partial x^{2}}, \quad c(x, t=0)=0, \quad c(L, t)=c_{\mathrm{pl}} .
$$

(3) For the metal hydride at $x \geq L, \frac{\partial c_{\mathrm{MH}}}{\partial x}=0$. As the rate of hydrogen absorbed in the metal hydride is defined as $R \equiv\left(L_{\mathrm{MH}}-L\right) \rho \frac{d c_{\mathrm{MH}}}{d t}$, the sorption rate is

$$
R=-\left.D \frac{\partial c}{\partial x}\right|_{x=L}
$$

The aim of this paper is to solve Eq. (22) and from that to calculate $R$ in Eq. (23). A general analytical time-dependent solution is not known. Valuable insight can, however, be gained from the analysis of representative special cases.

\section{B. Special cases: Time-dependent cases}

In Fig. 2 we have sketched the intermediate states before reaching a steady state in the diffusion layer. We show here that the time necessary to reach a steady state inside the diffusion layer is significantly shorter than that of the overall process. For this it is sufficient to consider the situation where $p_{\mathrm{pl}}=0$, which corresponds to $p \gg p_{\mathrm{pl}}$. The rate of $\mathrm{H}$ absorption is time dependent [Eq. (23)], as the surface is connected to the underlying metal hydride via the timedependent diffusion equation [Eq. (22)]. In the following, we will give its time dependence for three extreme cases, all with the assumption that the plateau pressure in the metal hydride is zero. This assumption is made to simplify the calculations.

Three cases are discussed:

(A) Dissociation-limited uptake-The dissociation barrier $E_{1}$ is large and thus $\theta \simeq 0 \Rightarrow c(x=0) \simeq 0$, with $s^{2} \simeq 1$. The redesorbed hydrogen, $b c^{2} \exp \left(-E_{2} / k T\right)$, is assumed to be negligible and the plateau pressure is low. Therefore the rate of dissociation determines the uptake rate:

$$
\frac{\partial c}{\partial x}=a e^{-E_{1} / k T} p \quad \text { at } x=0 \text { and } c(x=L, t)=c_{\mathrm{pl}} \simeq 0 .
$$

(B) Equilibrium of the surface with gas phase-The dissociation barrier is small. Here, the concentration of the outmost layer $c_{s}(p, T)$, which is given by Sievert's law, determines the rate:

$$
c(x=0, t)=c_{s} \Rightarrow s^{2}=\text { const and } c(x=L, t)=c_{\mathrm{pl}} \simeq 0 .
$$

(C) Chemisorption-limited uptake-The chemisorption enthalpy is large and with it $\theta \simeq 1$. Thus $g c$ is large: $\left(1-\frac{g c}{1+g c}\right)^{2} \simeq 0$, but not constant.

For case A one obtains ${ }^{27,28}$ 


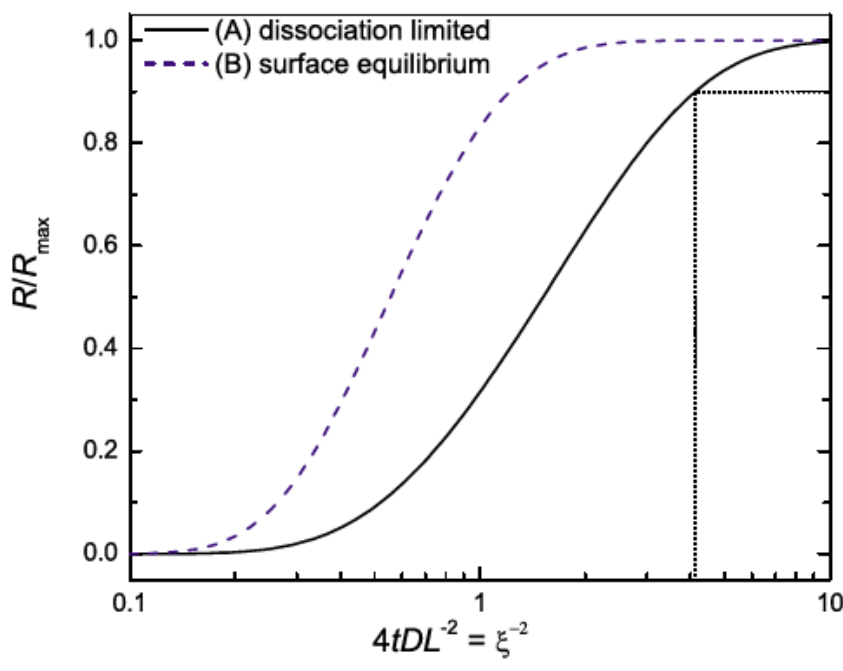

FIG. 4. (Color online) Time dependence of the hydrogen uptake, limited by dissociation (case A) and limited by the solubility of the surface layers (case B). The dotted line refers to the condition expressed in Eq. (26).

$$
R \propto 2 A p[\operatorname{erfc} \xi-\operatorname{erfc} 3 \xi+\operatorname{erfc} 5 \xi-\cdots] .
$$

For simplicity, we wrote $\xi=L / \sqrt{4 D t}$. Case B is modeled by ${ }^{27}$

$$
R \propto \frac{2 c_{s} \sqrt{D}}{\sqrt{\pi t}}\left[e^{-\xi^{2}}+e^{-3^{2} \xi^{2}}+e^{-5^{2} \xi^{2}}+\cdots\right] .
$$

The equations are numerically solved and the rate is shown in Fig. 4. For short times and small diffusion lengths, the rate is indeed time dependent for both cases. However, the rate saturates quickly. To discuss the relevance of the time dependence, we calculate the time after which more than $90 \%$ of the rate is reached:

$$
\frac{R(t)}{R_{\max }}>0.9 \text { for } t>\frac{L^{2}}{D} .
$$

Typical diffusion parameters are between $10^{-10} \mathrm{~m}^{2} / \mathrm{s}(\mathrm{Pd}$ at $300 \mathrm{~K})$ and $D=4.0 \times 10^{-13} \mathrm{~m}^{2} / \mathrm{s}(\mathrm{Mg}$ at $300 \mathrm{~K}) .{ }^{29}$ With typical diffusion lengths of around $10 \mathrm{~nm}=10^{-8} \mathrm{~m}$, one obtains diffusion times on the order of milliseconds. The time dependence is therefore negligible, as typical sorption cycles take several minutes. Therefore, the rate through a thin diffusion layer is in all cases time independent. In other words. it is impossible to distinguish between dissociation or diffusion processes on/in a thin cover layer via the time dependence of the total kinetics.

To include all possibilities, we have to solve case C. As considered in cases A and B, reaching a steady state in the diffusion layer is fast compared to the overall process. We neglect this time dependence and thus

$$
R \propto\left(1-\frac{g c}{1+g c}\right)^{2} a e^{-E_{1} / k T} p \propto p,
$$

as $c \propto c_{\mathrm{pl}}=$ const in steady state. The assumed constant concentration results from the constant chemical potential of the metal hydride at the plateau. Before reaching steady state, i.e., in the $\alpha$ phase of hydrogen in the metal hydride layer, a time dependence is expected, though: $c=c(t)$. This was experimentally observed in the $\mathrm{H}$ uptake in $\mathrm{Mg}$ films. ${ }^{30}$ The authors report decreasing hydrogen uptake in the $\alpha$ phase, where the chemical potential increases until the plateau has been reached. Unfortunately, the corresponding equations cannot be solved analytically anymore $\left[c(t) \propto \int R(t) d t\right]$. If a time dependence is observed, we can conclude that additional parameters, e.g., $p_{\mathrm{pl}}$ or $L$, change during the process. In this publication, we focus on the sorption kinetics in the plateau region, where $p_{\mathrm{pl}}$ and thus $s^{2}$ are time independent.

\section{Stationary case}

As estimated in Sec. II B, "steady state" is reached within short times compared to the time scale of a typical sorption process. The diffusion process can then be well described by a linear concentration gradient in the diffusion layer without time dependence. Thus

$$
\begin{aligned}
& A p-B c^{2}(0)=D \frac{c(0)-\alpha \sqrt{p_{\mathrm{pl}}}}{L} \equiv R, \\
& B c^{2}(0)+\frac{D}{L} c(0)-\frac{\alpha D}{L} \sqrt{p_{\mathrm{pl}}}-A p=0 .
\end{aligned}
$$

For simplification, we assume the self-blocking factor $s^{2}$ to be constant and include it in the parameters $A=s^{2} a e^{-E_{1} / k T}$ and $B=s^{2} b g^{2} e^{-E_{2} / k T}$. The surface is then coupled to the bulk via $\alpha=\sqrt{A / B}$ [from Eq. (20)]. The concentration under the surface, $c(0)$, is

$$
c(0)=\frac{-\frac{D}{L}+\sqrt{\left(\frac{D}{L}\right)^{2}+4 B\left(\frac{D}{L} \sqrt{\left.\frac{A}{B} p_{\mathrm{pl}}+A p\right)}\right.}}{2 B} .
$$

Finally, we obtain the rate

$$
R \equiv \frac{D}{L}\left[\frac{-\frac{D}{L}+\sqrt{\left(\frac{D}{L}\right)^{2}+4 B\left(\frac{D}{L} \sqrt{\frac{A}{B} p_{\mathrm{pl}}}+A p\right)}}{2 B}-\sqrt{\frac{A}{B} p_{\mathrm{pl}}}\right] .
$$

The role of the various parameters entering Eq. (31) is best discussed by means of its graphical representation given in Fig. 5. The left-hand side of Eq. (28) is an inverted parabola with a maximum at $p$; the right-hand side is a straight line with slope $D / L$. At equilibrium (i.e., $R=0$ and $p=p_{\mathrm{pl}}$ ), the two curves intersect the abscissa at $c_{\mathrm{pl}}=\alpha \sqrt{p_{\mathrm{pl}}}$. The concentration and rate $R$ are also determined by the intersection of the two functions in nonequilibrium, i.e., when $p \neq p_{\mathrm{pl}}$. For applied pressures below the equilibrium pressure $p_{\mathrm{pl}}$, the rates become negative (i.e., desorption occurs), and vice versa. However, the results are not point mirrored (Fig. 5). For a given value of $\frac{D_{1}}{L_{1}}$, the desorption rate $R_{1}$ (down pointing arrow) corresponds to the lowest applicable pressure (i.e., perfect vacuum) with $p=0$. For comparison the curve for an applied pressure of $p=2 p_{\mathrm{pl}}$ is also drawn (i.e., $\Delta p$ $=p_{\mathrm{pl}}$ in both cases). The absorption rate $R_{1}$ (down pointing arrow) is under no circumstances equal to that of desorption. 


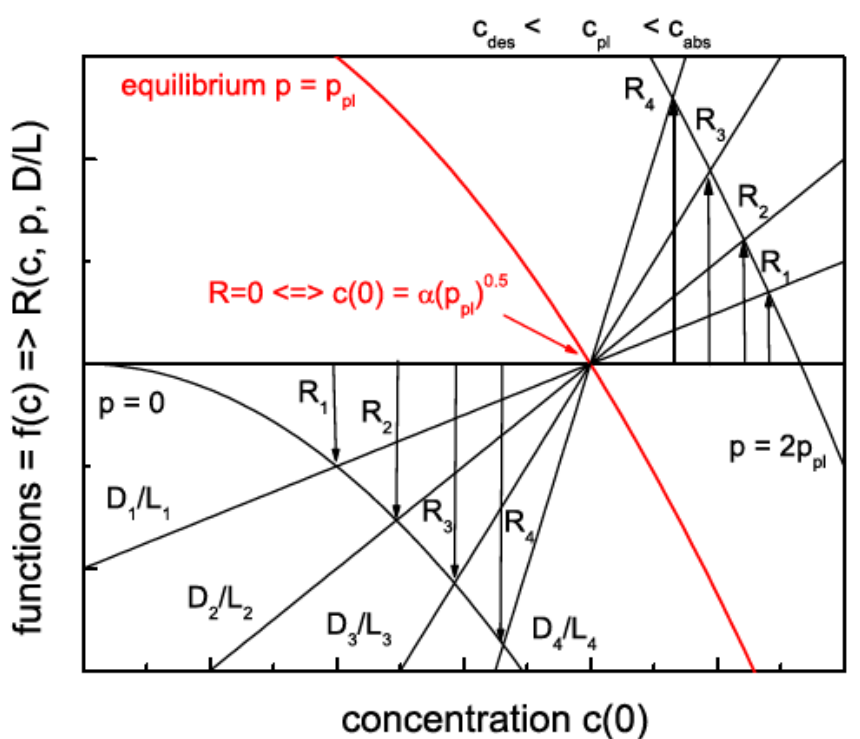

FIG. 5. (Color online) Graphical solution of Eq. (28) for various pressures and diffusion parameters $D_{4} / L_{4}>D_{3} / L_{3}>D_{2} / L_{2}$ $>D_{1} / L_{1}$, highlighting the interdependence of the uptake rate and the concentration at the surface.

Another source of asymmetry between hydrogen absorption and desorption is also due to the inherent hysteresis of the $p-c$ isotherms of any metal hydride (see Fig. 1). For the situation considered in the present paper, this means that $p_{\mathrm{pl}}$ during absorption is necessarily higher than $p_{\mathrm{pl}}$ during desorption and, consequently, that the desorption rate can be significantly lower than the absorption rate driven by the same pressure difference $\left|p-p_{\mathrm{pl}}\right|$. Another important point is that the surface concentration during absorption is inherently larger than that for desorption. This implies that a catalyst that affects the surface coverage will affect the sorption rate asymmetrically. If it is beneficial for absorption, it will negatively influence desorption and vice versa. Furthermore the absorption kinetics might be completely different form the desorption kinetics. This is well illustrated by the numerical and experimental results of Pasturel et al. ${ }^{31}$ who found that a diffusion layer with a high solubility (i.e., large $\alpha$ ) on top of a metal hydride supports desorption but hinders absorption. This result is in perfect agreement with Eq. (31), as the term $\alpha \sqrt{p_{\mathrm{pl}}}$ has to be as large as possible for desorption but as small as possible for absorption. It is noted, however, that the solubility leads to this result if the applied hydrogen pressure is $p \leq p_{\mathrm{pl}}$ or $p \simeq p_{\mathrm{pl}}$. For $p \gg p_{\mathrm{pl}} \simeq 0$, the behavior is reversed. Here, a high solubility accelerates the absorption kinetics. ${ }^{32}$ Hence, the effect of a specific parameter on the kinetics is relative. Therefore, we estimate Eq. (31) for various pressure regimes and relevant parameters.

We start with a Taylor series approximation of Eq. (31):

$$
\left.R(p)\right|_{p \approx p_{\mathrm{pl}}}=A \beta^{-1 / 2}\left(p-p_{\mathrm{pl}}\right)-2 A^{2} B \frac{L^{2}}{D^{2}} \beta^{-3 / 2}\left(p-p_{\mathrm{pl}}\right)^{2}+\cdots,
$$

with

$$
\beta=1+4 \frac{L}{D} \sqrt{A B p_{\mathrm{pl}}}+4 A B \frac{L^{2}}{D^{2}} p_{\mathrm{pl}} .
$$

For $A B p_{\mathrm{pl}} \ll D^{2} / L^{2}$, the kinetics is "dissociation limited," and the prefactor of the quadratic term is much smaller than that of the linear term. The kinetics can then be well described by

$$
R(p)=A^{\prime}\left(p-p_{\mathrm{pl}}\right),
$$

as frequently given in literature. ${ }^{33,34}$ The prefactor $A^{\prime} \simeq A$ involves an activation energy $E_{1}$. However, the apparent activation energy is different; see below.

The nonlinearity increases with larger pressures and/or decreasing diffusivity, i.e., $A p \gg D / L$. Eventually, this ends up in a square-root function,

$$
\left.R(p)\right|_{A p \gg D / L} \simeq \frac{D}{L} \alpha\left[\sqrt{p}-\sqrt{p_{\mathrm{pl}}}\right],
$$

in good agreement with the numerical results of Martin et $a l .{ }^{33}$ and Bloch. ${ }^{34}$ The surface parameters $A$ and $B$ have been replaced to demonstrate the similarity to the relation used to measure the hydrogen diffusion coefficient in permeation experiments. ${ }^{35}$ Equation (35) might be simplified to

$$
\left.R(p)\right|_{A p \gg D / L} \propto\left(k^{\prime} \frac{\sqrt{p}}{\sqrt{p_{\mathrm{pl}}}}-1\right) .
$$

Frequently, desorption is measured in or near vacuum conditions. In the very limit, Eq. (35) becomes

$$
R(p=0) \simeq-\frac{D}{L} \alpha \sqrt{p_{\mathrm{pl}}} \text { for } B \gg \frac{D}{L} .
$$

For the surface-limited kinetics, Eq. (31) is estimated by a Taylor series of the square root around $A p \simeq 0$ :

$$
R(p \simeq 0)=\frac{D^{2}}{2 B L^{2}}\left(-1+1+2 \gamma-2 \gamma^{2}-\frac{2 L}{D} \sqrt{A B p_{\mathrm{pl}}}\right),
$$

with

$$
\gamma^{2}=\left(\frac{L}{D} \sqrt{A B p_{\mathrm{pl}}}+A B p\right)^{2} \simeq A^{2} B \frac{L^{2}}{D^{2}} p_{\mathrm{pl}}+2 \frac{L}{D} p \sqrt{A^{3} B^{3} p_{\mathrm{pl}}} .
$$

This can be estimated for $B \ll \frac{D}{L}$ by using

$$
\left.R(p)\right|_{p \propto 0}=-A\left[p_{\mathrm{pl}}-p\right],
$$

and thus

$$
R(p=0) \simeq-A p_{\mathrm{pl}} \quad \text { for } B \ll \frac{D}{L} .
$$

It is convenient to use the reduced rate

$$
\left.R(p)\right|_{p \approx 0} \propto\left(k^{\prime \prime} \frac{p}{p_{\mathrm{pl}}}-1\right), \quad p<p_{\mathrm{pl}} .
$$

It is worth mentioning that the parameters $k^{\prime}$ and $k^{\prime \prime}$ in Eqs. (40) and (35) can be taken as a measure of the applicability of the model on the sorption kinetics. Per definition, $R\left(p=p_{\mathrm{p} 1}\right) \equiv 0$, and therefore $k^{\prime} \simeq 1 \simeq k^{\prime \prime}$. Deviations of $k^{\prime}$ and $k^{\prime \prime}$ from 1 means that even if the experimental fit was well 
TABLE I. Overview of the various estimates of the pressure dependence of Eq. (31) and the corresponding apparent activation energies.

\begin{tabular}{lcc}
\hline \hline \multicolumn{1}{c}{ Rate-limiting step condition } & Simplified kinetics & Activation energy \\
\hline Dissociation, $p \gg p_{\mathrm{pl}}$ & $R=A p$ & $E_{1}$ \\
Dissociation, $p \simeq p_{\mathrm{pl}}$ & $R=A\left(p-p_{\mathrm{pl}}\right)$ & $E_{1}+p_{\mathrm{pl}} / p-p_{\mathrm{pl}} 2 \Delta H_{\mathrm{MH}}$ \\
Diffusion, $p \gg p_{\mathrm{pl}}$ & $R=D / L \sqrt{A / B p}=D / L \alpha \sqrt{p}$ & $\Delta H_{\mathrm{sol}}+E_{\mathrm{diff}}-\sqrt{p}-\sqrt{p_{\mathrm{pl}}} \Delta H_{\mathrm{MH}}$ \\
Diffusion, $p \simeq p_{\mathrm{pl}}$ & $R=D / L \alpha\left[\sqrt{p}-\sqrt{p_{\mathrm{pl}}}\right]$ & $\Delta H_{\mathrm{sol}}+E_{\mathrm{diff}}+\sqrt{p_{\mathrm{pl}}} / \sqrt{p}-\sqrt{p_{\mathrm{pl}}}$ \\
Diffusion, $p_{\mathrm{pl}} \gg p \simeq 0$ & $R=-D / L \sqrt{A / B} \sqrt{p_{\mathrm{pl}}}=D / L \alpha \sqrt{\mathrm{diff}}-\Delta H_{\mathrm{MH}}$ \\
Recombination, $p_{\mathrm{pl}} \gg p \simeq 0$ & $R=-A p_{\mathrm{pl}}$ & $E_{1}-2 \Delta H_{\mathrm{MH}}$ \\
\multicolumn{1}{c}{ Rate-limiting step condition } & Best-fit kinetics & Activation energy \\
\hline Dissociation & $R \propto\left(k^{\prime \prime} p / p_{\mathrm{pl}}-1\right)$ & \\
Diffusion & $R \propto\left(k^{\prime} \sqrt{p} / \sqrt{p_{\mathrm{pl}}}-1\right)$ & \\
\hline \hline
\end{tabular}

adapted on a local scale, an interpretation of the values by physical parameters would be problematic.

The activation energy is a complicated function of pressures and temperature. The activation energy is calculated from Eq. (1): $E_{\mathrm{AA}}=k T^{2} d \ln R / d T$. Six extreme cases can be distinguished: (i) and (ii), where plateau pressure $p_{\mathrm{pl}} \ll p$, which corresponds to absorption at very high pressures; (iii) and (iv), sorption near the plateau, where $p \simeq p_{\mathrm{pl}}$; and (v) and (vi), where $p_{\mathrm{pl}} \gg p$, corresponding to desorption at low pressures $(p \simeq 0)$ or high temperatures $\left(p_{\mathrm{pl}} \gg p>0\right)$. Each pair of cases has one case where dissociation is dominant and another where diffusion is dominant. We have

(i) $E_{\mathrm{AA}}=E_{1}$ for $p \gg p_{\mathrm{pl}}$ and $A B p_{\mathrm{pl}} \ll D^{2} / L^{2}$,

(ii) $E_{\mathrm{AA}}=\frac{1}{2}\left(E_{1}-E_{2}\right)+E_{\mathrm{diff}}$

$$
\text { for } p \gg p_{\mathrm{pl}} \text { and } A B p_{\mathrm{pl}} \gg D^{2} / L^{2} \text {, }
$$

in agreement with our previous paper. ${ }^{32}$ With a heat of hydride formation $\Delta H_{\mathrm{MH}}$, the plateau pressure is $p_{\mathrm{pl}}$ $\propto e^{2 \Delta H_{\mathrm{MH}} / k T}$, and the apparent activation energies of the rate become

$$
\begin{aligned}
& \text { (iii) } E_{\mathrm{AA}}=E_{1}+\frac{p_{\mathrm{pl}}}{p-p_{\mathrm{pl}}} 2 \Delta H_{\mathrm{MH}} \\
& \text { for } p \simeq p_{\mathrm{pl}} \text { and } A B p_{\mathrm{pl}} \ll D^{2} / L^{2}, \\
& \text { (iv) } \Delta H_{\mathrm{sol}}+E_{\mathrm{diff}}+\frac{\sqrt{p_{\mathrm{pl}}}}{\sqrt{p}-\sqrt{p_{\mathrm{pl}}}} \Delta H_{\mathrm{MH}} \\
& \text { for } p \simeq p_{\mathrm{pl}} \text { and } A B p_{\mathrm{pl}} \gg D^{2} / L^{2} .
\end{aligned}
$$

These intermediate solutions diverge at $p=p_{\mathrm{pl}}$. Cases (v) and (vi) are derived via the Taylor approximation [Eq. (37)]. The apparent activation energies become

$$
\text { (v) } E_{\mathrm{AA}}=E_{1}-2 \Delta H_{\mathrm{MH}} \text { for } p_{\mathrm{pl}} \gg p \text { and } \frac{D}{L} \gg B \text {, }
$$

and

(vi) $E_{\mathrm{AA}}=\Delta H_{\mathrm{sol}} E_{\mathrm{diff}}-\Delta H_{\mathrm{MH}}$ for $p_{\mathrm{pl}} \gg p$ and $\frac{D}{L} \ll B$.

The equations resemble the Temkin equation ${ }^{36,37}$ if a reaction $A \rightarrow B$ with one single activation barrier $E_{\mathrm{AB}}$ is coupled to an equilibrium state $C$ with stability $\Delta H_{C}$ :

$$
A \leftarrow m B \rightleftharpoons m C, \quad E_{\mathrm{AA}}=E_{\mathrm{AB}}-m \Delta H_{C} .
$$

For the diffusion-limited case, atomic hydrogen $(\mathrm{H})$ diffuses inside the metal hydride $\mathrm{MH}_{x}$, i.e., $m=1$ :

$$
\left(\frac{1}{2} H_{2} \leftarrow\right) H_{\text {chem }} \leftarrow H_{\text {diff }} \rightleftharpoons \mathrm{MH}_{x} .
$$

For the surface-limited case, two atomic hydrogen recombine at the surface to $\mathrm{H}_{2}$, i.e., $m=2$ :

$$
H_{2} \leftarrow 2 H_{\text {chem }}\left(\leftarrow 2 H_{\text {diff }}\right) \rightleftharpoons 2 \mathrm{MH}_{x} .
$$

The change in the parameter $m$ corresponds to a transition from a diffusion-limited to a surface-limited sorption. Thus, $r=m / 2$ is also called reaction order. ${ }^{37}$ This is in special cases reflected by the pressure dependence of the rates. A linear pressure dependence corresponds to a surface-limited kinetics; a square-root dependence corresponds to a diffusionlimited kinetics. This is discussed in detail in Sec. III B. To highlight the various cases and to simplify the discussion, Table I gives an overview.

\section{EXPERIMENTAL EXAMPLES}

Hydrogen-uptake kinetics have been measured for a huge number of hydride systems. We restrict ourselves to representative examples, the $\mathrm{H}$ uptake in thin films, the sorption kinetics of bulk $\mathrm{LaNi}_{5} \mathrm{H}_{6}$, and $\mathrm{Mg}-\mathrm{MgH}_{2}$. The first example 
resembles the geometry of the theoretical model, and is thus ideal for testing the model. The applicability of the model is demonstrated by the representative real-world hydrogen storage materials $\mathrm{LaNi}_{5} \mathrm{H}_{6}$ and $\mathrm{MgH}_{2}$. For $\mathrm{LaNi}_{5}$, the model is only approximately valid; the good agreement between anticipated and measured values confirms the usability of the model in general, though. For the last example, the model is applied to the $\mathrm{Mg}-\mathrm{MgH}_{2}$ system, which shows an archetypical "diffusion-limited" growth.

\section{A. Thin film samples: Kinetics and pressure dependence of the sorption kinetics of $\mathrm{Mg}_{y} \mathrm{Ni}_{1-y}$}

Hydrogen switchable mirrors resemble the setup of the model perfectly. The hydride material is deposited on a glass substrate, on which a Pd capping layer is grown. In good approximation, we can speak of a surface layer, consisting of the surface-active material (Pd) and a diffusion layer (Pd and hydride are separated by a thin oxide layer). In this thin-film system, the sorption kinetics is studied as a function of composition, thickness, temperature, and hydrogen pressure (see, e.g., Ref. 32). The technique can be extended to a combinatorial screening method by the use of large area thin-film matrix samples with controlled gradients in local chemical composition of two or more constituents. ${ }^{38}$

To measure the hydrogen-sorption kinetics in thin films quantitatively, the optical transmission in $\mathrm{Mg}_{y} \mathrm{Ni}_{1-y}$ films is monitored during hydrogen loading. The structural phase transitions of the system are accompanied by changes in the electronic structure, affecting the optical properties of the material. ${ }^{39}$ It is found that the hydride grows from the substrate interface for composition around $\mathrm{Mg}_{4} \mathrm{Ni}$, which is hint for a very fast diffusion of in the metal. As described in detail in Ref. 38, the hydrogen-uptake kinetics of these films can be determined from the optical intensity changes.

The hydrogen loading behavior of the samples is monitored by exposing the whole sample to hydrogen gas in an optical setup. Technical details are described elsewhere. ${ }^{40}$ Figure 6 shows the pressure dependence of $\mathrm{H}$ absorption of $\mathrm{Mg}_{y} \mathrm{Ni}_{1-y}$ thin films for various compositions $y$ measured at room temperature. The gradient film is completely covered with a uniform layer of $\mathrm{Pd}$, making it likely that we are dealing with the same surface properties. ${ }^{41}$ An increased amount of $\mathrm{Mg}$ leads in first order to a decrease in the diffusivity of $\mathrm{H}$ in $\mathrm{Mg}_{y} \mathrm{Ni}_{1-\gamma}$. Furthermore, the formed phases change from a $\mathrm{Mg}_{2} \mathrm{NiH}_{4} / \mathrm{MgH}_{2}$ mixture to a $\mathrm{Ni}$-doped $\mathrm{MgH}_{2} \cdot{ }^{38}$ This has consequences for the thermodynamical properties, in particular for the equilibrium pressures. The plateau pressures are obtained as extrapolation to zero rate using expression (34) based on the model derived above (see Fig. 6). The heat of formation is obtained by repeating the measurements at various temperatures. ${ }^{38}$ The method holds also for nonlinear kinetics (Mg-rich samples; see inset of Fig. 6), although the extrapolation to zero rate becomes more difficult.

Here, we emphasize that measuring a linear pressure dependence does not mean that the uptake rate is determined by surface properties only since they are equal for all compositions on a given gradient sample: Although the sample is
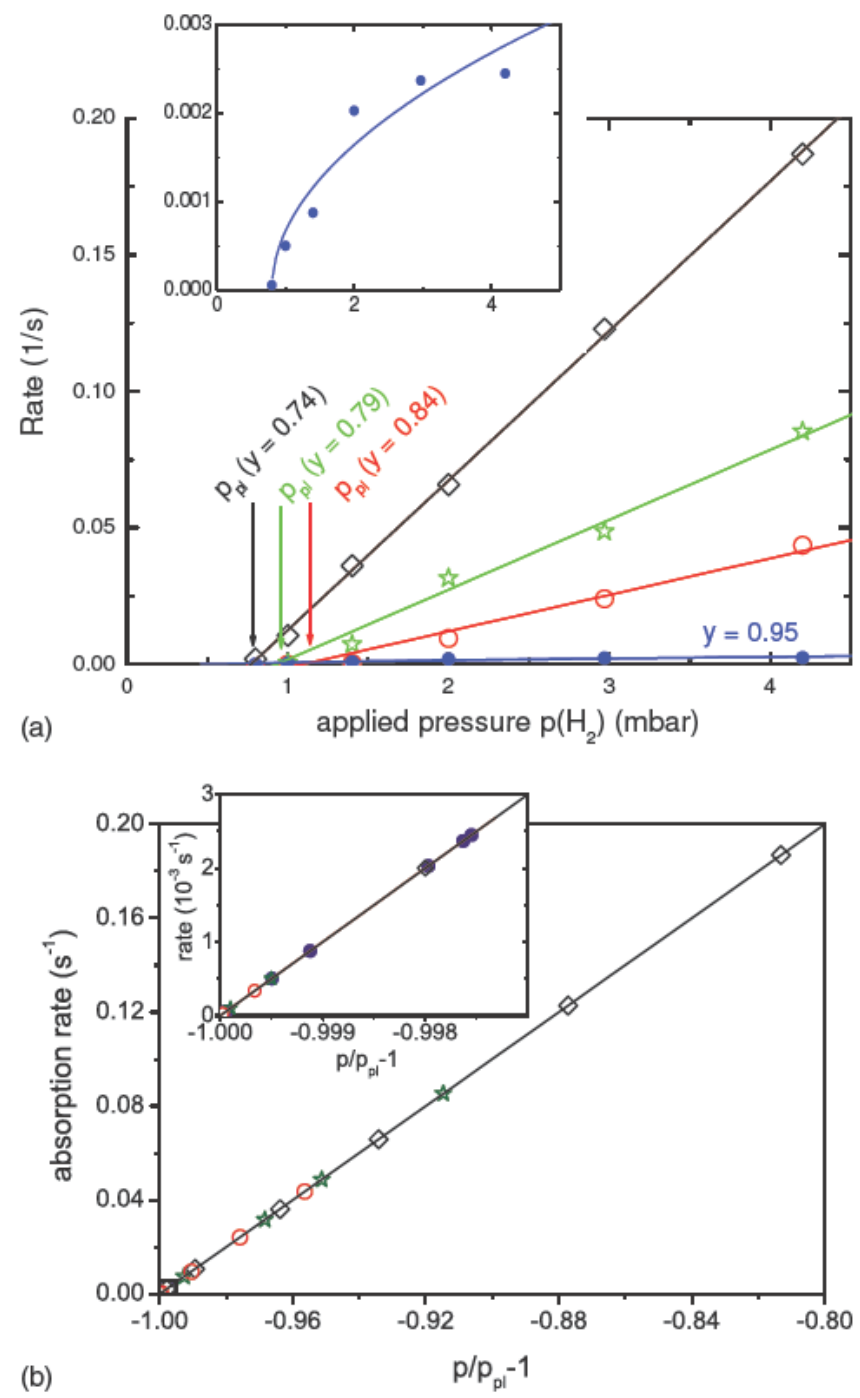

FIG. 6. (Color online) (a) Pressure dependence of $\mathrm{H}$ absorption of $\mathrm{Mg}_{1-y} \mathrm{Ni}_{y}$ thin films for various compositions $y$ measured at room temperature $[y=0.74$ (black) open diamonds; 0.79 (green) stars; 0.84 (red) empty circles; 0.95 (blue) filled circles]. The solid lines are linear fits, from which the plateau pressures are derived. The regression coefficient decreases from nearly 1 (0.9997, black dots) to 0.9011 (blue dots). The inset is an enlargement of the $\mathrm{Mg}$ rich sample, which clearly shows the nonlinear pressure dependence. The extrapolation to zero rate defines the equilibrium pressure $p_{\mathrm{pl}}$. Data were from Ref. 38. (b) The plateau pressures from (a) are used to plot the rate as a function of $p / p_{\mathrm{pl}}$. The solid curves are linear fits to the experimental data, revealing the same slope for all curves.

covered with $\mathrm{Pd}$ of the same thickness and all compositions are probed simultaneously under the same conditions, still a large difference in uptake kinetics is measured. This can be easily understood as the linear relation [Eq. (34)] is a firstorder approach. Evidently, the rates are determined by one rate-limiting step only in extreme cases. Most of the rates can be reasonably fitted by a linear relation, although the linearity of the data decreases (decreasing of the correlation coefficient; see Fig. 6), which is an indication of the increasing influence of the bulk. A purely diffusion-limited case is not found either. To underline the statement, we plot the rates 
as a function of $\left(p / p_{\mathrm{pl}}-1\right)$ [Eq. (40)]. Linear fits to the experimental data reveal the same slope for all curves. Evidently, the uptake rate can be well described by Eq. (40) with the same proportionality parameter, i.e., with the same properties of the surface layer (Pd). It is noted that the deviation from 0 for $p / p_{\mathrm{pl}}$ of the Mg-richest sample is the smallest of all samples (see inset of Fig. 6). Thus, the linear approximation is still very well suited, even if a different behavior is to be expected at higher supersaturation.

The interdependence of elementary steps solves a controversial debate: How can the change in bulk properties (e.g., diffusion) influence the uptake rate, which was considered to be "surface rate limited" on the basis of a traditional single step by an evaluation of the time dependence ${ }^{42}$ or pressure dependence ${ }^{43}$ of the desorption of $\mathrm{MgH}_{2}$ ? In our model, this controversy does not arise. The coverage at the surface $\theta$ is directly connected to the bulk concentration $c(x=0)$ at the surface, which depends on the diffusion gradient in the volume, which further depends on the plateau pressure of the absorbing material. The surface coverage, however, determines the hydrogen dissociation (parameters $A$ and $B$ ), and thus a bulk process influences a surface process.

We can also discuss the validity of the simplifications of the two-step model along this experimental example. It is not expected that the absolute value of $\theta$ changes significantly in the used pressure range (between 1 and $4 \mathrm{mbar}$ ), and thus the simplification in Eq. (28) is plausible. Furthermore, the results are in good agreement with literature data. The hydrogen uptake of $\mathrm{Mg}$ thin films in the $\alpha$ regime shows a timedependent behavior, while the absorption in the plateau regime is time constant. ${ }^{30}$

An important outcome of the two-step model is that the apparent activation energy depends on the exact thermodynamic regime, in which this property is measured. To validate this experimentally, we measured optically the hydrogen-uptake rate of a Pd-capped $\mathrm{Mg}_{2} \mathrm{Ni}$ film at various temperatures and pressures. The uptake rate is plotted as a function of the applied pressure in Fig. 7. The linear behavior as already discussed above is a hint for a dissociation-limited rate. The extrapolated onset pressures represent the equilibrium pressures. From the temperature dependence, a heat of hydride formation of $-44 \mathrm{~kJ} / \mathrm{mol} \mathrm{H}_{2}$ is obtained as shown by the corresponding van't Hoff plot in Fig. 8. The linear extrapolation to higher temperatures of the thin-film values matches exactly the equilibrium pressures obtained from bulk $p c T$ measurements. ${ }^{39,44}$ This underlines the applicability of the method. Without considering any models, the apparent activation energy is calculated according to Eq. (1) from the change in the rate at two different temperatures:

$$
E_{\mathrm{AA}}=k \frac{1}{4}\left(T_{1}+T_{2}\right)^{2} \frac{\ln R_{2}-\ln R_{1}}{T_{2}-T_{1}} .
$$

At a given pressure, e.g., 40 mbar [marked with (1) in Fig. 7], the rate decreases with increasing temperature. The corresponding apparent activation energy is thus negative. At higher pressures, though, the activation energy is positive [point (2)]. The reason for this is obvious due to the different thermodynamic driving forces $\left(p-p_{\mathrm{eq}}\right)$. Assuming

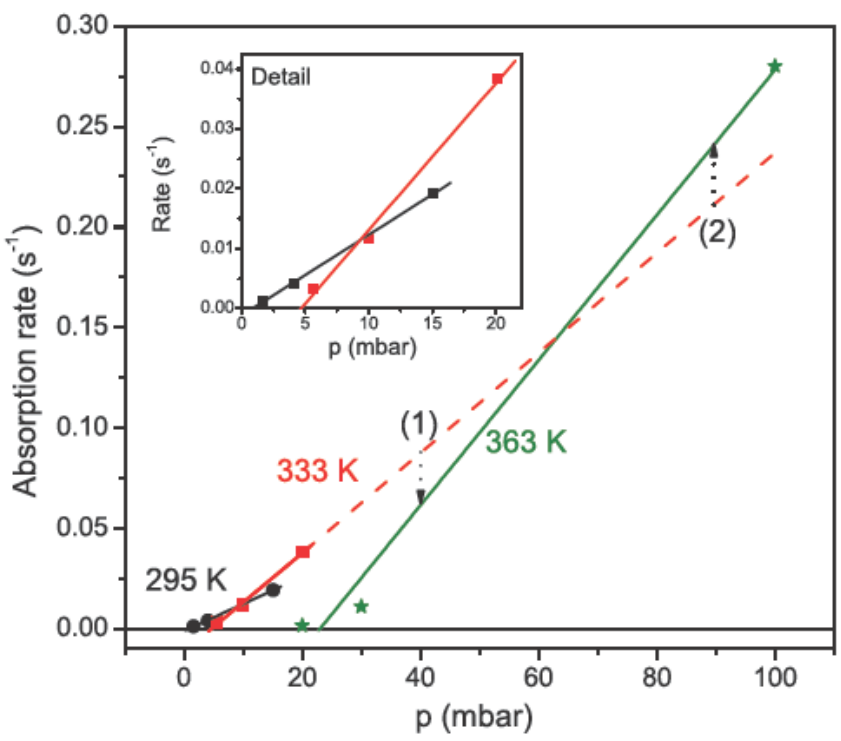

FIG. 7. (Color online) $\mathrm{H}$-uptake rate of $\mathrm{Mg}_{2} \mathrm{Ni}$ at various pressures and temperatures measured optically. Points marked (1) and (2) indicate pressure regimes, where a negative and a positive apparent activation energy, respectively, are found (see text for details).

dissociation-limiting kinetics, the apparent activation energy is zero at

$$
\frac{-E_{1}}{2 \Delta H_{\mathrm{MH}}}=\frac{p_{\mathrm{eq}}}{p-p_{\mathrm{eq}}} .
$$

The intercepts of the curves in Fig. 7 correspond to zero apparent activation energy and are found around $p / p_{\mathrm{eq}}=4$. The surface-barrier height $E_{1}=0.16 \mathrm{eV}$ is obtained from an Arrhenius plot of the parameter $A^{\prime}$ [from Eq. (32)], shown in Fig. 9. Using this value, a zero apparent activation energy is expected at $p / p_{\mathrm{eq}}=4$ and is indeed found.

The experimental surface-barrier height of $0.16 \mathrm{eV}$ is in good agreement with the value for hydrogen dissociation on

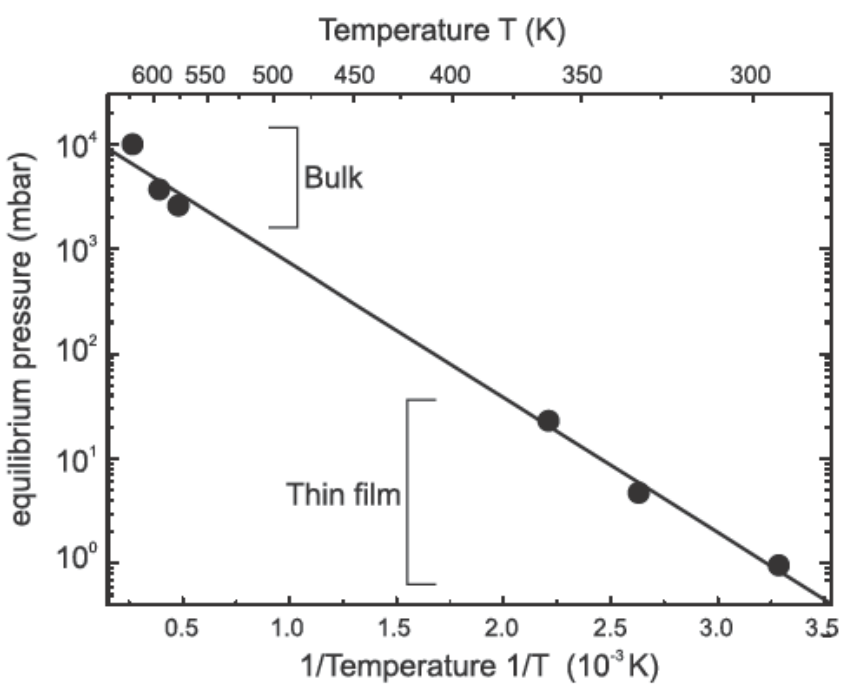

FIG. 8. van't Hoff plots of $\mathrm{Mg}_{2} \mathrm{Ni}$. Equilibrium pressures were from Fig. 7 (thin films) and Ref. 44 (bulk). 


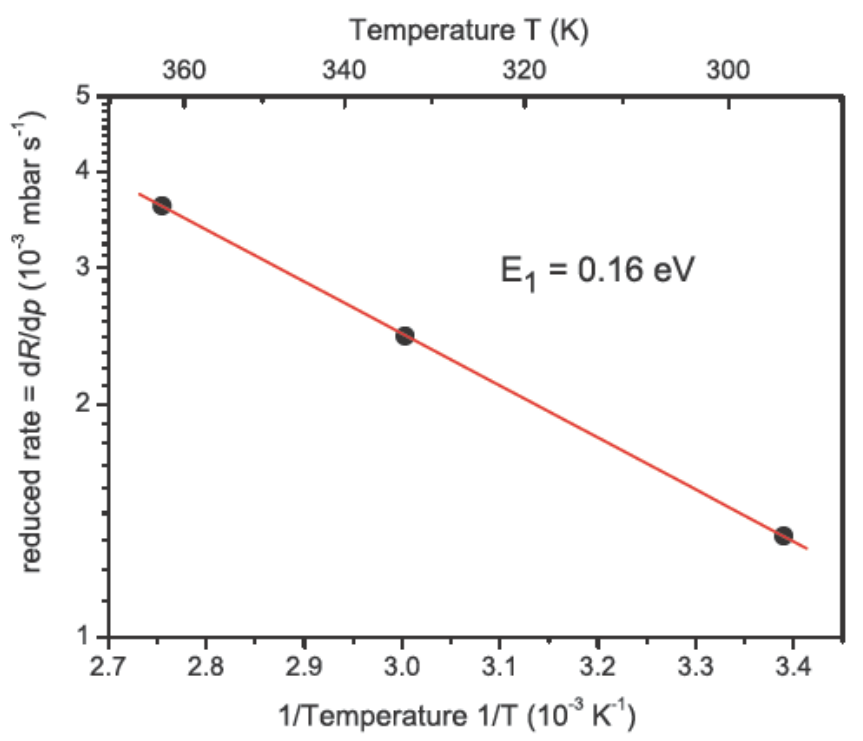

FIG. 9. (Color online) Surface activation energy $E_{1}$ of Pdcapped $\mathrm{Mg}_{2} \mathrm{Ni}$ thin films as obtained from an Arrhenius plot of the parameter $A^{\prime}=R /\left(p-p_{\mathrm{pl}}\right)$ [Eq. (32)].

Pd as derived from surface science results. Hydrogen dissociation on Pd surfaces is nonactivated, i.e., $E_{\text {diss }} \leq 0$. However, at higher coverage, the chemisorbed hydrogen changes the electronic structure (poisoning), ${ }^{45}$ which shifts the energy potential by around $0.2 \mathrm{eV}$. The experimental activation energy of hydrogen absorption in pure Pd was found to be 0.2 $\mathrm{eV}$, but the authors did not distinguish between surface and diffusion activation energy. ${ }^{35}$

\section{B. Thin film samples: Activation energy of hydrogen absorption in metal-coated thin films}

Metal coatings on metal hydrides are frequently used to improve hydrogen-uptake kinetics in thin films. These are optimum model systems to validate the presented continuum model. Two data sets are described in detail: (i) hydrogen uptake in metal-coated Ta wires (from Ref. 46) and (ii) hydrogen uptake in metal-coated surface-oxidized yttrium films (from Ref. 32). Hydrogen diffusion is very fast in Ta with a low diffusion barrier $\left(E_{\text {diff }}=0.136 \mathrm{eV}\right)$ at high solubility $\left(\Delta H_{\text {sol }}=-0.4 \mathrm{eV} / \mathrm{H}\right) .{ }^{47}$ The main barrier is thus the surface layer (dissociation and diffusion in the metal coating). Hydrogen diffusion is comparably fast in yttrium. ${ }^{48}$ Here the main diffusion barrier is the surface oxide layer separating metal coating and yttrium $\left[E_{\mathrm{diff}} \simeq 1.3 \mathrm{eV}\right.$, estimated from the related systems $\mathrm{TiO}_{2}, E_{\text {diff }}=0.59-1.3 \mathrm{eV},{ }^{49}$ and $\mathrm{Al}_{2} \mathrm{O}_{3}$, $E_{\text {diff }}=1.0 \mathrm{eV}$ (Ref. 50)]. Coating of these materials with $d$ metals can increase the $\mathrm{H}$ uptake significantly as shown in Fig. 10. Interestingly, the apparent activation energy of yttrium films decreases with decreasing rate, i.e., it shows the compensation effect, while that of Ta wires increases with decreasing rate. The compensation effect is a hint of a change in rate-limiting process. We will demonstrate this quantitatively on the yttrium thin-film results, as the boundary conditions are well suited for simplifying the reaction kinetics. We will show that the results on Ta wires also con-

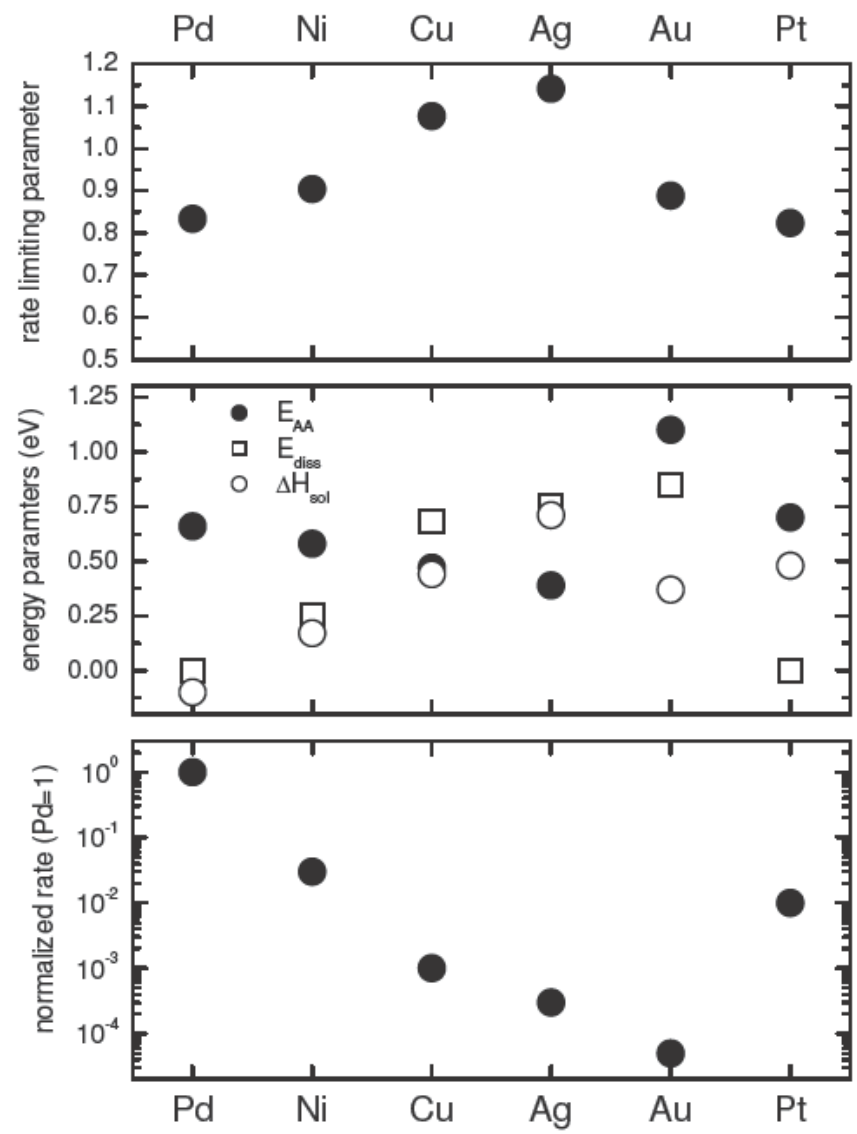

FIG. 10. Bottom panel: Hydrogen-uptake rates of $d$ metalcoated Ta wires (hollow dots) and of surface-oxidized yttrium films (full dots). Middle panel: Corresponding apparent activation energies and the dissociation barrier heights and activation energies for permeation through the metal coating. Top panel: rate limiting parameter $r$ as derived from Eq. (51).

firm the model qualitatively. Yttrium dihydride is very stable, and with an applied hydrogen pressure of 2 bars, $p \gg p_{\mathrm{pl}}$ $\simeq 0.013$ bar at $T \simeq 250{ }^{\circ} \mathrm{C} .51$

With $p \gg p_{\mathrm{pl}}$, the apparent activation energy is given by

$$
E_{\mathrm{AA}}=2 E_{\mathrm{diff}}-E_{2}+k T^{2} \frac{d}{d T} \ln \left(\sqrt{1+4 \frac{A B L^{2} p}{D^{2}}}-1\right) .
$$

With $\zeta=\frac{4 A B L^{2} p}{D^{2}}$ we obtain

$$
E_{\mathrm{AA}}=2 E_{\text {diff }}-E_{2}+\left(E_{1}+E_{2}-2 E_{\text {diff }}\right) \frac{\frac{1}{2} \zeta}{\begin{array}{c}
(1+\zeta) \pm \sqrt{1+\zeta} \\
=r
\end{array}} .
$$

The factor $r=\frac{1}{2} m$ defines the regime of the system. It varies between $\frac{1}{2}$ for diffusion-limited rate $\left(D^{2} \ll 4 A L^{2} B p\right.$, i.e., $\zeta$ $\rightarrow \infty)$ and 1 for $\zeta=0$, i.e., for a surface-limited rate. The energy parameters are $E_{2}=E_{1}-2 \Delta H_{\text {sol }}$ and $E_{1}=E_{\text {diss. }}$. The apparent activation energies become 


$$
E_{\mathrm{AA}}=2 E_{\mathrm{diff}}-E_{\mathrm{diss}}+2 \Delta H_{\mathrm{sol}}+r\left[2 E_{\mathrm{diss}}-2 \Delta H_{\mathrm{sol}}-2 E_{\mathrm{diff}}\right] .
$$

This delivers the activation energy of permeation in the diffusion-limited case,

$$
r=\frac{1}{2}: E_{\mathrm{AA}}=E_{\mathrm{diff}}+\Delta H_{\mathrm{sol}},
$$

and the activation energy of dissociation in the dissociationlimited case,

$$
r=1: E_{\mathrm{AA}}=E_{\mathrm{diss}},
$$

as calculated in Eq. (41). Figure 10 compares the measured apparent activation energies with $E_{\text {diss }}$ and $E_{\text {diff }}+\Delta H_{\text {sol }}$-apparently without good agreement. Thus we use Eq. (50) to evaluate $r$ (see Fig. 10, top). The parameter varies from around 0.75 to 1 , clearly demonstrating the change from a diffusion-limited to a dissociation-limited regime.

We can calculate the pressure dependence by using

$$
\frac{d \ln R}{d p_{\mathrm{H}_{2}}}=\frac{d \ln R}{d \zeta} \frac{d \zeta}{d p_{\mathrm{H}_{2}}}=\frac{1}{p_{\mathrm{H}_{2}}} r
$$

Thus

$$
r=\frac{d \ln R}{d \ln p} .
$$

That means that the reaction order $r$ can also be determined by the pressure dependence of the absorption rate. The reaction rate $r$ of Pd obtained by this method is $r=0.8$, in good agreement with the value derived from the temperature dependence of the rate. ${ }^{32}$

A similar treatment of the data on Ta wires is difficult. The plateau of tantalum hydride is not very pronounced and $p_{\mathrm{pl}}$ lies in the same range of the applied hydrogen pressure at the used temperatures. The above used assumptions do not hold; thus a calculation of the reaction order according to Eq. (51) is not possible. The authors measure a square-root dependence of the pressure dependence of the uptake of Pdcovered Ta wires and a diffusion-limited rate is thus likely. The measured apparent activation energies follow roughly the activation energy of permeation. This is in agreement with the measured dependence on the layer thickness $R$ $\propto 1 / L$, though a Cu-covered Ta-wire shows a different behavior. Furthermore, the authors measured an activation energy, which depends on $L$. The apparent activation energy of $\mathrm{Ni}$-coated Ta wires decreases with increasing thickness $L$. Simultaneously the rate decreases, which is clear evidence for the compensation effect. The disagreement of measured and estimated activation energies is therefore due to a change in reaction order.

It is useful to calculate the effect of $L$ on $r$. From Eq. (51) it follows that

$$
r \simeq 1-\frac{\alpha}{4}=1-\frac{k_{L} L^{2}}{4} \quad \text { for } \zeta \rightarrow 0,
$$

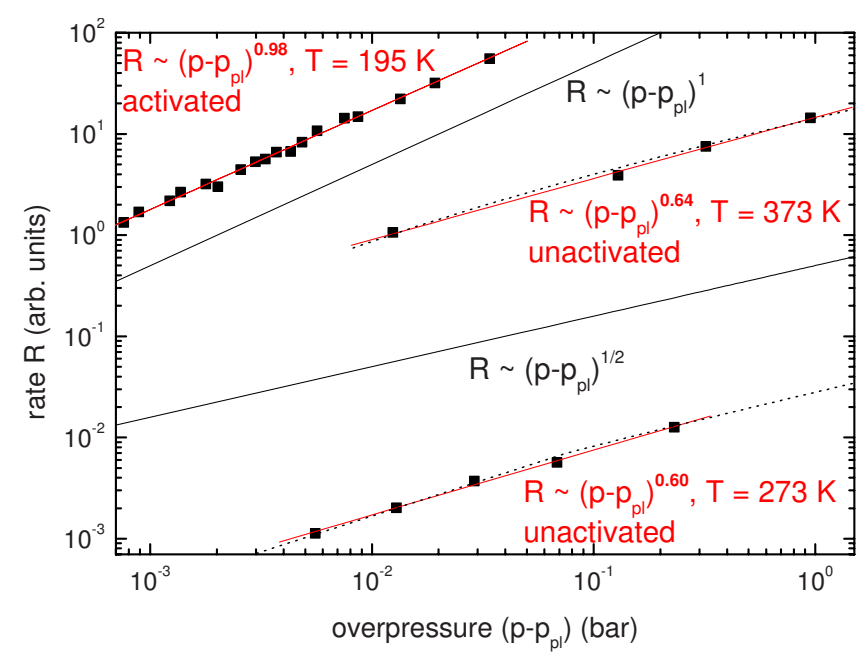

FIG. 11. (Color online) Pressure dependence of the $\mathrm{H}$ absorption of activated and unactivated $\mathrm{LaNi}_{5} \mathrm{H}_{x}$ at various temperatures. Data were from Refs. 53 and 54. The data are fitted to Eq. (31) (dashed curves) and to Eq. (58) (full curves).

$$
r \simeq \frac{1}{2 \sqrt{\zeta}}+\frac{1}{2}=\frac{1}{2 k_{L}^{\prime} L}+\frac{1}{2} \quad \text { for } \zeta \rightarrow \infty .
$$

$k_{L}$ and $k_{L}^{\prime}$ are constants. Clearly, the rate is limited by diffusion $\left(r=\frac{1}{2}\right)$ in the overlayer only if $L \rightarrow \infty$. Conversely, for zero-layer thickness, only the surface barrier limits the uptake $(r=1)$. It is noted, though, that the reaction order concept holds only for negligible thermodynamic influence (i.e., $\left.p / p_{\mathrm{pl}} \gg 1\right)$. In the following, we apply this model on bulk results.

\section{Powder samples: $\mathrm{LaNi}_{5} \mathrm{H}_{x}$}

$\mathrm{LaNi}_{5}$ is well known to form an oxide layer on top of each grain. This oxide layer hinders hydrogen dissociation as well as hydrogen diffusion, whereas diffusion in $\mathrm{LaNi}_{5}$ is very fast. ${ }^{52}$ This system can thus well be described by the twostep model. Experimentally, the initial absorption rates for activated $\mathrm{LaNi}_{5}$ at $195 \mathrm{~K}$ and for unactivated $\mathrm{LaNi}_{5}$ at two different temperatures are shown in Fig. 11 (data from Refs. 53 and 54). The rates for activated $\mathrm{LaNi}_{5}$ were found to be dependent upon $\Delta p=p-p_{\mathrm{pl}}$. The rates were found to be independent of the total $\mathrm{H}: \mathrm{LaNi}_{5}$, the ratio along the twophase coexistence region, provided that they were compared at the same value of $\Delta p$. The pressure dependence is different from that for unactivated $\mathrm{LaNi}_{5}$, in the region of primary solubility, for which it was experimentally found to be approximately $p^{1 / 2}$ (here, the plateau pressure is set to be $\simeq 0$ ). The striking contradiction between these two results is that activated $\mathrm{LaNi}_{5}$ is expected to have better surface properties (i.e., diffusion-limited kinetics) than the unactivated material. ${ }^{55}$ Ergo the pressure for the activated sample should be $p^{1 / 2}$ rather than linear as observed, which raised a controversial debate on the effect. ${ }^{54}$ Our explanation is relatively straightforward. Absorption in unactivated $\mathrm{LaNi}_{5}$ is hindered by the thin oxide layer on the metal grains. ${ }^{55}$ The observed pressure dependence can be explained if the rate-limiting 
step is diffusion in this layer. ${ }^{56}$ After activation, this oxide layer has been removed, and the dissociation/recombination of hydrogen is rate limiting. We would like to emphasize that in no case an exponent of 1 or $1 / 2$, respectively, is found. "Rate limiting" has thus to be understood in the sense of "dominating the kinetics."

This was investigated in detail by Uchida. ${ }^{57}$ At pressures well above the plateau pressure (see table), the reaction kinetics can be modeled by ${ }^{57}$

$$
R=R_{0} p^{r},
$$

where $R_{0}$ is a proportional constant and $r$ is an exponent of pressure (Temkin equation $m=2 r$ ), and can be interpreted as the reaction order defined in Eq. (50). Uchida ${ }^{57}$ measured the reaction kinetics of initial hydrogen absorption in $\mathrm{LaNi}$ alloys. He found values for $r$ to be between 0.5 and 1 . $r$ becomes close to 0.5 ( $\mathrm{H}$ permeation through the surface layers) with $E_{\mathrm{AA}}>15 \mathrm{~kJ} / \mathrm{mol}$, which were measured for the heavily oxidized samples with grown oxide layers at temperatures lower than $300 \mathrm{~K}$. $r$ becomes close to $1\left(\mathrm{H}_{2}\right.$ dissociation) with $E_{\mathrm{AA}}<5 \mathrm{~kJ} / \mathrm{mol}$, which were measured for the samples with thin oxide layers at temperatures higher than $700 \mathrm{~K}$. These results indicate the shift of the rate-controlling step of the $\mathrm{H}_{2}$-absorption rate between $\mathrm{H}$ permeation and $\mathrm{H}_{2}$ dissociation, depending upon the surface conditions. Moreover, the results obey the Constable-Cremer relation (high rate at high apparent activation energies) - in good agreement with the results from Andreasen et al. ${ }^{12}$ The data points in Fig. 11 have been fitted to the two-step model [Eq. (31)] with empirical parameters. The change in the parameters underlines the above given explanation: The change in the diffusion parameters is $D_{u} / L_{u} / D_{a} / L_{a}=1 / 860$ (the indices $u$ and $a$ correspond to unactivated and activated) and that of the surface parameters $\sqrt{\frac{A_{u}}{B_{u}} / \frac{A_{a}}{B_{a}}}=\frac{1}{500}$. Even though both processes are improved, the increase in the surface parameters is less than those in the diffusion parameters, leading to a "surfacedominated" kinetics.

Furthermore, Boser ${ }^{2}$ proposed that the slow step for both absorption and desorption of hydrogen in the two-phase region is the phase transition. The reported energy of activation was found to be similar to the enthalpy change for the transition from the hydrogen-saturated metal to the hydride phase. This explanation cannot explain the observed kinetics nor the pressure dependence. Interestingly, the value of $E_{\mathrm{AA}}$ is in very good agreement with our model. For diffusionlimited kinetics (and similarly for dissociation-limited one), the heat of hydride formation enters the activation energy (see Table I). Because the dissociation barrier and the diffusion barrier heights are very small in $\mathrm{LaNi}_{5}$, the activation energy resembles mainly the heat of hydride formation $\left[\Delta H\left(\mathrm{LaNi}_{5} \mathrm{H}_{5.5}\right)=-15 \mathrm{~kJ} / \mathrm{mol} \mathrm{H}\right.$ (Ref. 58)].

\section{Powder samples: Oxide-catalyzed $\mathrm{Mg}-\mathrm{MgH}_{2}$}

The mathematical description implies that the rate of absorption and desorption should be time constant irrespective of the particular rate-limiting step. However, most experimentally measured kinetics do show a time-dependent rate. In this case, the given parameters change during the sorption

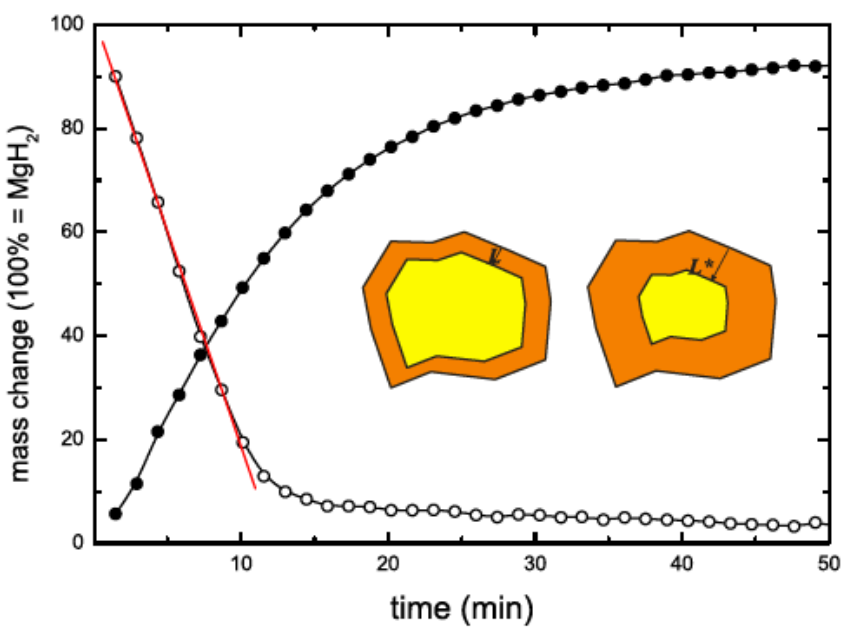

FIG. 12. (Color online) Absorption and desorption kinetics of ball-milled $\mathrm{MgH}_{2}$. For desorption into vacuum and absorption at 5 bar $\mathrm{H}_{2}$, the temperature is in both cases $300{ }^{\circ} \mathrm{C}$. The absorption behavior is best described by a three-dimensional controlled contracting volume $(L \rightarrow L *)$ model with decreasing interface velocity (i.e., $d L / d t \neq$ const).

process. The corresponding kinetics of an archetypical example of ball-milled $\mathrm{Mg}-\mathrm{MgH}_{2}$ sorption are shown in Fig. 12.

While the desorption curve can be fitted with a linear function and therefore shows a nearly constant rate, the absorption behavior is clearly nonlinear. The absorption behavior is best described by the three-dimensional diffusioncontrolled contracting volume model with decreasing interface velocity, ${ }^{42,59}$ which is sketched in Fig. 12. After a an initial nucleation state, a dense $\mathrm{MgH}_{2}$ is formed at the surface of a $\mathrm{Mg}$ grain. ${ }^{21,60} \mathrm{MgH}_{2}$ has very low diffusional properties and is therefore a major barrier for hydrogen transport ( $\mathrm{H}$ permeation in $\mathrm{Mg}$ is faster by a factor of 1000 at room temperature). ${ }^{29,61} \mathrm{H}$ uptake in $\mathrm{Mg}$ corresponds to the growth of the hydride layer, which means that the diffusion path length is increasing, too. Thus, the uptake rate is time dependent ("decreasing interface velocity"). During desorption, hydrogen has to diffuse through $\mathrm{Mg}$ with relatively high hydrogen diffusivity. Desorption kinetics is thus mainly controlled by the surface (i.e., linear kinetics). ${ }^{62-64}$ Liang et al. ${ }^{43}$ found a linear pressure dependence of desorption in the form of Eq. (40) with $k^{\prime \prime} \neq 1$ without being able to assign this behavior to a specific mechanism. Our model suggests that in this case the kinetics is not purely limited by a single step (i.e., dissociation).

Additives attached to the surface can enhance the sorption kinetics, interestingly with the same effect both for absorption and desorption. ${ }^{42,65}$ This is in sharp contrast to a single rate-limiting step, while this situation can be perfectly described by the (one-dimensional) two-layer model. The surface of $\mathrm{MgH}_{2}$ consists of a thin $\mathrm{MgO}$ layer, which has a high dissociation barrier. Moreover, the hydrogen coverage can be assumed to be low. The chemical potential is different from those in the previously discussed cases. Here, diffusion takes place in the solubility range of the hydride (previously of the oxide layer), and the chemical potential in the metal layer, 

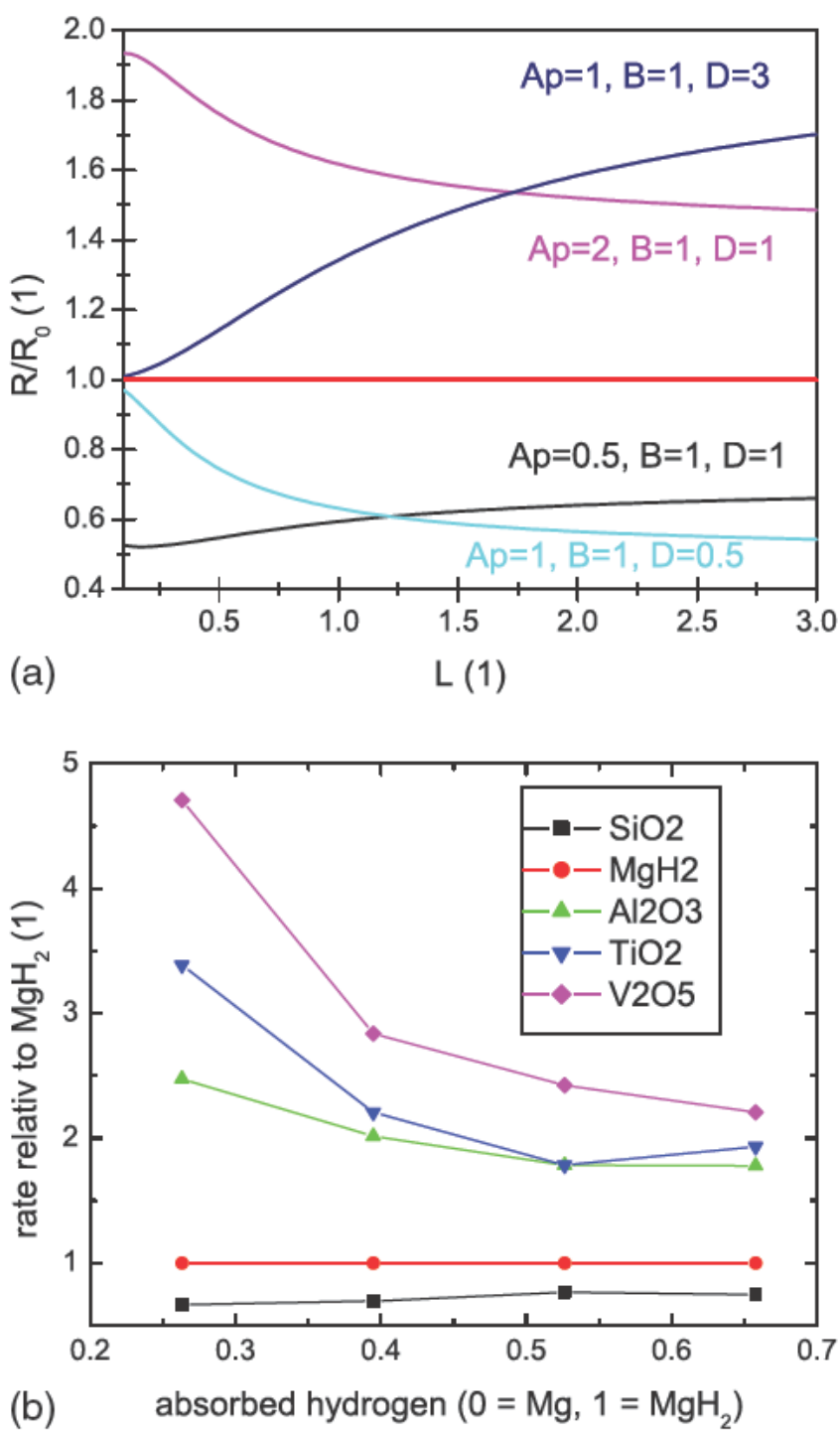

FIG. 13. (Color online) Impact $R / R_{0}$ of additives on the absorption properties of metal hydrides. (a) Dependence of the impact on the diffusion path length $L$ as simulated by the two-step model with model parameters for diffusion and surface parameters $A$ and $B$. (b) The effect of various additives on the absorption kinetics relative to that of as-milled $\mathrm{MgH}_{2}$ is plotted as a function of the filling factor. Data were taken from Ref. 65.

here $\mathrm{Mg}$, remains constant as does the concentration, because the $\mathrm{H}$ uptake is connected to the growth of the diffusion layer (i.e., $\mathrm{MgH}_{2}$, with thickness $L$; diffusion in the oxide is neglected). The concentration at the (moving) interface is constant $\left(\Rightarrow c_{\mathrm{pl}}\right)$. Therefore the mathematics of the two-step model can be used to describe this completely different hydride growth mode. However, the rate is not constant anymore, because $L=L(t)$, and with a change in $L$ the relevance of the surface to bulk changes. The last piece of information gives us a hint to test the assumption of a multistep situation: The impact of the additives on diffusion is negligible at initial state and increases with increasing $L$; the impact on the surface properties is strongest at initial state. This is plotted for model parameters in Fig. 13 (top panel).
In three-dimensional (3D) structures, the rate is not strictly proportional to the growth speed of the diffusion layer. Still, the filling factor of a grain $\left(0=\mathrm{Mg}, 1=\mathrm{MgH}_{2}\right)$ is an approximate measure of the diffusion layer thickness $L$. The effect of various additives on the absorption kinetics relative to that of as-milled $\mathrm{MgH}_{2}$ is plotted as a function of the filling factor in Fig. 13 (bottom panel). Obviously, the additive affects absorption mainly at initial states. Even the effect of $\mathrm{SiO}_{2}$, which slows down the absorption kinetics, is reproduced by our model. All additives affect mainly the surface parameter $A$, although a mainly diffusion-controlled kinetics is observed.

\section{SUMMARY AND CONCLUSIONS}

Recent measurements of activation energies of various hydrogen storage materials posed intriguing questions about their physical meaning. In order to rationalize the experimental results, a one-dimensional model is set up to estimate the time, temperature, and pressure dependences of sorption kinetics and the relevance of several materials parameters involved in the process. The model, which incorporates explicitly surface and diffusion processes, results in three main conclusions:

(1) It is impossible to distinguish between dissociation and diffusion processes on/in a thin cover layer via the time dependence of the total kinetics.

(2) In most cases, a single rate-limiting step does not exist.

(3) The apparent activation energy and the pressure dependence of the rate are functions of the relative importance of the elementary processes involved in $\mathrm{H}$ sorption and $E_{\mathrm{AA}}$ is thus not an intrinsic materials property.

The conclusions are in good agreement with published experimental results. For example, the time dependence of diffusion as investigated in Ref. 27 agrees well with our results as do the pressure relations of the rates. The pressure dependence of the activation energy was also reported in Refs. 66 and 67, which is hint that the apparent activation energy is not a materials property in general. Our model considers the interdependence of surface and diffusion processes and can thereby calculate the apparent activation energy from intrinsic materials properties. However, the estimates of the pressure and temperature dependencies for extreme cases are the same as those obtained by assuming single rate-limiting steps (see, e.g., Refs. 33 and 34).

The model is successfully tested for several experimental cases, ranging from model systems (thin films) to practical systems ( $\mathrm{LaNi}_{5} \mathrm{H}_{x}$ and $\mathrm{MgH}_{2}$ powder samples). Thin films have the advantage of having the same geometry as our onedimensional model and having well-defined surface properties. With thin films it is possible to demonstrate experimentally the interdependence of surface and bulk processes. The mathematical description of practical hydrogen storage materials is complicated by their 3D morphology. Nevertheless, the main results from the present one-dimensional model remain valid, as for example demonstrated by the good description for the pressure dependence of the H-absorption kinetics of $\mathrm{LaNi}_{5}$ bulk. We showed the applicability of the 
model to the sorption kinetics of $\mathrm{Mg}-\mathrm{MgH}_{2} \cdot{ }^{20} \mathrm{Here}$, the geometry is a function of time change. However the principles of this paper can be applied, e.g., pressure dependence. The model sheds light on the functioning of additives in $\mathrm{MgH}_{2}$, which are found to enhance the surface properties also for a diffusion-limited kinetics.

Can we make use of these findings to, e.g., optimize hydrogen storage materials? One interesting fact is that a change in many parameters (except $E_{1}$ and $D$ ) improving absorption or desorption can worsen the reversed reaction. One example described by Pasturel et al. ${ }^{31}$ is the increase in the solubility of the diffusion layer, which improves desorption but worsens absorption. The same holds for the surface coverage, which in the presented model is described by the parameters $a$ and $b$.

The present model is applicable to the sorption kinetics of "classical" metal hydrides, which is mainly determined by dissociation and diffusion of hydrogen. In complex hydrides, additional elementary steps have to be included. Still, there is experimental evidence that also their thermodynamics and kinetics are strongly interdependent. ${ }^{68} \mathrm{We}$ are planning to extend our model to include these cases.

*andreas.borgschulte@empa.ch

${ }^{1}$ L. Schlapbach and A. Züttel, Nature (London) 414, 353 (2001); G. W. Crabtree, M. Dresselhaus, and M. Buchanan, Phys. Today 57(12), 39 (2004).

${ }^{2}$ O. J. Boser, J. Less-Common Met. 46, 91 (1976).

${ }^{3}$ M. Miyamoto, K. Yamaji, and Y. Nakata, J. Less-Common Met. 89, 111 (1983).

${ }^{4}$ M. J. Ron, J. Alloys Compd. 283, 178 (1999).

${ }^{5}$ Z. Haberman, J. Bloch, M. H. Mintz, and I. Jakob, J. Alloys Compd. 253-254, 556 (1997).

${ }^{6}$ A. Osovizky, J. Bloch, M. H. Mintz, and I. Jakob, J. Alloys Compd. 245, 168 (1996).

${ }^{7}$ A. Borgschulte, R. J. Westerwaal, J. H. Rector, B. Dam, and R. Griessen, Appl. Phys. Lett. 85, 4884 (2004).

${ }^{8}$ B. Hammer and J. Norskov, Nature (London) 376, 238 (1995).

${ }^{9}$ T. Vegge, Phys. Rev. B 70, 035412 (2004).

${ }^{10}$ M. Tsuda, W. Dino, H. Kasai, H. Nakanishi, and H. Aikawa, Thin Solid Films 509, 157 (2006).

${ }^{11}$ K. Nobuhara, H. Kasai, W. A. Dino, and H. Nakanishi, Surf. Sci. 566-568, 703 (2004).

${ }^{12}$ A. Andreasen, T. Vegge, and A. S. Pedersen, J. Phys. Chem. B 109, 3340 (2005).

${ }^{13}$ F. H. Constable, Proc. R. Soc. London, Ser. A 108, 355 (1925).

${ }^{14}$ G. C. Bond, Appl. Catal., A 191, 23 (2000).

${ }^{15}$ T. Bligaard, K. Honkala, A. Logadottir, J. K. Norskov, S. Dahl, and C. J. H. Jacobsen, J. Phys. Chem. B 107, 9325 (2003).

${ }^{16}$ See, e.g., W. J. Moore, Physical Chemistry (Addison Wesley, Longman, Harlow England, 1972), p. 360, and references therein.

${ }^{17}$ W. E. Wallace, R. F. Karlicek, and H. Imamura, J. Phys. Chem. 83, 1708 (1979).

${ }^{18}$ P. Nieto, E. Pijper, D. Barredo, G. Laurent, R. A. Olsen, E.-J. Baerends, G.-J. Kroes, and D. Farías, Science 312, 86 (2006).

${ }^{19}$ F. Faglioni and W. A. Goddard, J. Chem. Phys. 122, 014704 (2005).

${ }^{20}$ A. Borgschulte, M. Bielmann, A. Zuettel, G. Barkhordarian, M. Dornheim, and R. Bormann, Appl. Surf. Sci. 254, 2377 (2008).

${ }^{21}$ V. P. Zhdanov, A. Krozer, and B. Kasemo, Phys. Rev. B 47, 11044 (1993).

${ }^{22}$ T. Mitsui, M. K. Rose, E. Fomin, D. F. Ogletree, and M. Salmeron, Nature (London) 422, 705 (2003)

${ }^{23}$ D. Tomanek, S. Mukherjee, V. Kumar, and K. H. Bennemann, Surf. Sci. 114, 11 (1982); See, also, D. McLean, Grain Boundaries in Metals (Oxford University Press, London, 1957).

${ }^{24}$ T. McMullen, M. J. Stott, and E. Zaremba, Phys. Rev. B 35, 1076 (1987).

${ }^{25}$ N. P. Serdyuk and A. L. Chuprina, Russ. J. Phys. Chem. 54, 1615 (1980).

${ }^{26}$ The factor of 2 results from different units: $\left[E_{i}\right]=\mathrm{eV} / \mathrm{H}_{2}$ and $\left[\Delta H_{\mathrm{sol}}\right]=\mathrm{eV} / \mathrm{H}$.

${ }^{27}$ J. Crank, The Mathematics of Diffusion (Clarendon, Oxford, 1975).

${ }^{28}$ H. D. Baehr and K. Stephan, Wärme- und Stoffübertragung (Springer, Berlin, 2004), p. 268.

${ }^{29}$ J. Renner and H. J. Grabke, Z. Metallkd. 69, 639 (1978).

${ }^{30}$ M. Johansson, C. W. Ostenfeld, and I. Chorkendorff, Phys. Rev. B 74, 193408 (2006).

${ }^{31}$ M. Pasturel, R. J. Wijngaarden, W. Lohstroh, H. Schreuders, M. Slaman, B. Dam, and R. Griessen, Chem. Mater. 19, 624 (2007).

${ }^{32}$ A. Borgschulte, J. H. Rector, H. Schreuders, B. Dam, and R. Griessen, J. Catal. 239, 263 (2006)

${ }^{33}$ M. Martin, C. Gommel, C. Borkhart, and E. Fromm, J. Alloys Compd. 238, 193 (1996).

${ }^{34}$ J. Bloch, J. Alloys Compd. 312, 135 (2000).

${ }^{35} \mathrm{E}$. Wicke and H. Brodowsky, in Topics in Applied Physics: Hydrogen in Metals II, Application Oriented Properties, edited by G. Alefeld and J. Voelkl (Springer-Verlag, Berlin, 1978).

${ }^{36}$ M. I. Temkin, Dokl. Akad. Nauk SSSR 152, 156 (1963); Int Chem. Eng. 11, 709 (1971).

${ }^{37}$ P. W. Atkins, Physical Chemistry (Oxford University Press, Oxford, 1994).

${ }^{38}$ R. Gremaud, C. P. Broedersz, D. M. Borsa, A. Borgschulte, P. Mauron, H. Schreuders, J. H. Rector, B. Dam, and R. Griessen, Adv. Mater. (Weinheim, Ger.) 19, 2813 (2007).

${ }^{39}$ W. Lohstroh, R. J. Westerwaal, J. L. M. van Mechelen, C. Chacon, E. Johansson, B. Dam, and R. Griessen, Phys. Rev. B 70, 165411 (2004)

${ }^{40}$ A. Borgschulte, W. Lohstroh, R. J. Westerwaal, H. Schreuders, J. H. Rector, B. Dam, and R. Griessen, J. Alloys Compd. 404-406, 699 (2005).

${ }^{41}$ This assumption is approximate. Due to morphology, which depends on the composition of $\mathrm{Mg}_{1-y} \mathrm{Ni}_{y}$, the surface properties of the capping layer are also composition dependent. See A. Borgschulte, R. Gremaud, S. de Man, R. J. Westerwaal, J. H. Rector, B. Dam, and R. Griessen, Appl. Surf. Sci. 253, 1417 (2006).

${ }^{42} \mathrm{G}$. Barkhordarian, T. Klassen, and R. Bormann, J. Alloys Compd. 407, 249 (2006). 
${ }^{43}$ G. Liang, J. Huot, S. Boily, A. Van Neste, and R. Schulz, J. Alloys Compd. 291, 295 (1999).

${ }^{44}$ J. J. Reilly and R. H. Wiswall, Inorg. Chem. 7, 2254 (1968).

${ }^{45}$ N. Lopez, Z. Lodziana, F. Illas, and M. Salmeron, Phys. Rev. Lett. 93, 146103 (2004). The authors considered ideal surfaces, but the effect will be even enhanced on practical surfaces, i.e., with steps, impurities, etc.

${ }^{46}$ K. Nakamura, H. Uchida, and E. Fromm, J. Less-Common Met. 80, P19 (1981).

${ }^{47}$ Y. Fukai, The Metal-Hydrogen System, Basic Bulk Properties, Springer Series in Materials Science Vol. 21 (Springer, Berlin, 1993) p. 207.

${ }^{48}$ S. J. van der Molen, J. W. J. Kerssemakers, J. H. Rector, N. J. Koeman, B. Dam, and R. Griessen, J. Appl. Phys. 86, 6107 (1999).

${ }^{49}$ O. W. Johnson, S. H. Paek, and J. W. Deford, J. Appl. Phys. 46, 1026 (1975).

${ }^{50}$ E. Serra, A. C. Bini, G. Cosoli, and L. Pilloni, J. Am. Ceram. Soc. 88, 15 (2005).

${ }^{51}$ L. N. Yannopoulos, R. K. Edwards, and P. G. Wahlbeck, J. Phys. Chem. 69, 2510 (1965).

${ }^{52}$ C. Schönfeld, R. Hempelmann, D. Richter, T. Springer, A. J. Dianoux, J. J. Rush, T. J. Udovic, and S. M. Bennington, Phys. Rev. B 50, 853 (1994).

${ }^{53}$ S. Tanaka, J. D. Clewley, and T. B. Flanagan, J. Phys. Chem. 81, 1684 (1977).

${ }^{54}$ S. Tanaka, J. D. Clewley, and T. B. Flanagan, J. Less-Common Met. 56, 137 (1977).

${ }^{55}$ L. Schlapbach, Hydrogen in Intermetallic Compounds, edited by L. Schlapbach (Springer-Verlag, Berlin, 1992), Vol. II.
${ }^{56}$ W. J. Dunning, in Kinetics of Reactions in Ionic Systems, Materials Science Research Vol. 4, edited by T. J. Gray and V. D. Frechette (Plenum, New York, 1969), p. 132.

${ }^{57}$ H. Uchida, Int. J. Hydrogen Energy 24, 861 (1999).

${ }^{58} \mathrm{R}$. Griessen and T. Riesterer, in Hydrogen in Intermetallic Compounds, edited by L. Schlapbach (Springer-Verlag, Berlin, 1988), Vol. I.

${ }^{59}$ M. H. Mintz and Y. Zeiri, J. Alloys Compd. 216, 159 (1995).

${ }^{60}$ G. Friedlmeier, and M. Groll, J. Alloys Compd. 253-254, 550 (1997).

${ }^{61}$ J. Töpler, H. Buchner, H. Säufferer, K. Knorr, and W. Prandl, J. Less-Common Met. 88, 397 (1982).

${ }^{62}$ K. Bohmhammel, B. Christ, and G. Wolf, Thermochim. Acta 310, 167 (1998).

${ }^{63}$ J. F. Fernandez and C. R. Sanchez, J. Alloys Compd. 356-357, 348 (2003).

${ }^{64}$ F. H. Ellinger, C. E. Holley, Jr., B. B. McInteer, D. Pavone, R. M. Potter, E. Staritzky, and W. H. Zachariasen, J. Am. Chem. Soc. 77, 2647 (1955).

${ }^{65}$ W. Oelerich, T. Klassen, and R. Bormann, J. Alloys Compd. 315, 237 (2001).

${ }^{66}$ H. N. Murty, D. L. Biederman, and E. A. Heintz, J. Phys. Chem. 72, 746 (1968).

${ }^{67}$ P. S. Rudman, J. Appl. Phys. 50, 7195 (1979).

${ }^{68} \mathrm{LiBH}_{4}$, P. Mauron, F. Buchter, O. Friedrichs, A. Remhof, M. Bielmann, C. N. Zwicky, and A. Zuttel, J. Phys. Chem. B 112, 906 (2008); $\mathrm{NaAlH}_{4}$, W. Luo and K. J. Gross, J. Alloys Compd. 385, 224 (2004). 